\title{
Pharmacological treatments of COVID-19
}

\author{
Adeleh Sahebnasagh ${ }^{1} \cdot$ Razieh Avan $^{2} \cdot$ Fatemeh Saghafi $^{3} \cdot$ Mojataba Mojtahedzadeh $^{4} \cdot$ Afsaneh Sadremomtaz $^{5}$. \\ Omid Arasteh $^{6} \cdot$ Asal Tanzifi $^{7,8} \cdot$ Fatemeh Faramarzi $^{9} \cdot$ Reza Negarandeh $^{10} \cdot$ Mohammadreza Safdari $^{11}$. \\ Masoud Khataminia ${ }^{12} \cdot$ Hassan Rezai Ghaleno ${ }^{13}$. Solomon Habtemariam ${ }^{14} \cdot$ Amirhosein Khoshi $^{15}$
}

Received: 9 April 2020 / Revised: 31 July 2020 / Accepted: 10 August 2020 / Published online: 20 August 2020

(c) Maj Institute of Pharmacology Polish Academy of Sciences 2020

\begin{abstract}
The viral infection due to the new coronavirus or coronavirus disease 2019 (COVID-19), which was reported for the first time in December 2019, was named by the World Health Organization (WHO) as Severe Acute Respiratory SyndromeCoronavirus-2 (SARS-CoV2), because of the very similar genome and also its related symptoms to SARS-CoV1. The ongoing COVID-19 pandemic with significant mortality, morbidity, and socioeconomic impact is considered by the WHO as a global public health emergency. Since there is no specific treatment available for SARS-CoV2 infection, and or COVID-19, several clinical and sub-clinical studies are currently undertaken to find a gold-standard therapeutic regimen with high efficacy and low side effect. Based on the published scientific evidence published to date, we summarized herein the effects of different potential therapies and up-to-date clinical trials. The review is intended to help readers aware of potentially effective COVID-19 treatment and provide useful references for future studies.
\end{abstract}

Keywords COVID-19 $\cdot$ Pharmacology $\cdot$ Pneumonia $\cdot$ SARS-CoV2

Electronic supplementary material The online version of this article (https://doi.org/10.1007/s43440-020-00152-9) contains supplementary material, which is available to authorized users.

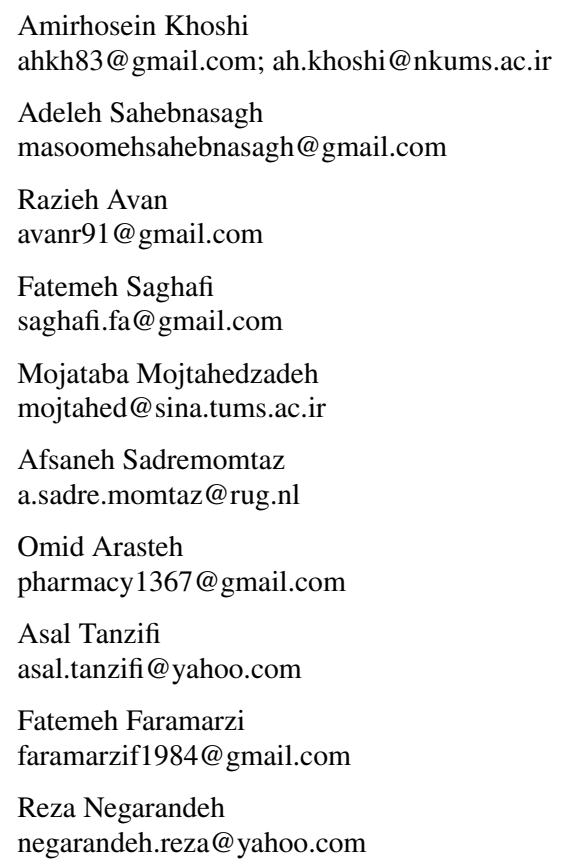

Mohammadreza Safdari
safdari57@yahoo.com

Masoud Khataminia masoodkhataminia@yahoo.com

Hassan Rezai Ghaleno Hassan_Rezai2020@yahoo.com

Solomon Habtemariam s.habtemariam@herbalanalysis.co.uk

1 Clinical Research Center, Department of Internal Medicine, North Khorasan University of Medical Sciences, Bojnurd, Iran

2 Department of Clinical Pharmacy, Medical Toxicology and Drug Abuse Research Center (MTDRC), Faculty of Pharmacy, Birjand University of Medical Sciences, Birjand, Iran

3 Department of Clinical Pharmacy, Faculty of Pharmacy and Pharmaceutical Sciences Research Center, Shahid Sadoughi University of Medical Sciences, Yazd, Iran

4 Department of Clinical Pharmacy, Pharmaceutical Sciences Research Center, Tehran University of Medical Sciences, Tehran, Iran 


\section{Introduction}

In the first two decades of the twenty-first century alone, three pandemic infectious diseases caused by the human coronaviruses (CoVs) have emerged: Severe Acute Respiratory Syndrome Coronavirus-1 (SARS-CoV1), Middle East Respiratory Syndrome coronavirus (MERS-CoV), and the now, a novel coronavirus (SARS-CoV2 or nCoV-19). In the latter case, the outbreak was identified first in December 2019 in Wuhan city, the capital of Hubei Province, China [1-3]. The transmission rate of nCoV-19 in humans appears to be higher than SARS-CoV1 and MERS-CoV [4]. The Coronaviridae Study Group (CSG) of the International Committee on Taxonomy of Viruses (ICTV) renamed $\mathrm{nCoV}-19$ as SARS-CoV2 [5], while the disease was named by the World Health Organization (WHO) as Coronavirus Disease-2019 (COVID-19) [6]. Based on the latest WHO report, the number of SARS-CoV2 cases (over 6.4 million globally with more than 379 thousand mortalities, updated on 2 June 2020) will undoubtedly be counted by the millions.

Structurally, coronaviruses have a positive-sense RNA genome with $26-32 \mathrm{~kb}$ range in length which is surrounded by nucleocapsid and envelope [6]. Two-thirds of the viral RNA is mainly located in the first open-reading frame (ORF), including ORF1a/b, and translates ppla and pp1ab

5 XB20 Drug Design, Groningen Research Institute of Pharmacy, University of Groningen, 9700 AD Groningen, The Netherlands

6 Department of Clinical Pharmacy, School of Pharmacy, Mashhad University of Medical Sciences, Mashhad, Iran

7 Sepanta Faragene Azma Research Laboratory. Co. LTD., Gorgan, Iran

8 Department of Parasitology, Faculty of Medicine, Mazandaran University of Medical Sciences, Sari, Iran

9 Clinical Pharmacy Research Center, Iran University of Medical Sciences, Tehran, Iran

10 Student Research Committee, Department of Pharmaceutics, Faculty of Pharmacy, Mazandaran University of Medical Sciences, Sari, Iran

11 Department of Orthopedic Surgery, Faculty of Medicine, North Khorasan University of Medical Sciences, Bojnurd, Iran

12 Student Research Committee, Faculty of Pharmacy, Tehran University of Medical Sciences, Tehran, Iran

13 Department of Surgery, Faculty of Medicine, Lorestan University of Medical Sciences, Khorramabad, Iran

14 Pharmacognosy Research Laboratories and Herbal Analysis Services, University of Greenwich, Central Avenue, Chatham-Maritime, Kent ME4 4TB, UK

15 Department of Clinical Biochemistry, School of Medicine, North Khorasan University of Medical Sciences, Arkan roadway, Bojnurd, Iran polyproteins, and also encodes different non-structural proteins (NSP), which are key enzymes in the viral life cycle [7, 8]. Several accessory proteins, which interfere with the host innate immune response, and also four essential structural proteins, including spike (S) glycoprotein, small envelope (E) protein, matrix $(\mathrm{M})$ protein, and nucleocapsid $(\mathrm{N})$ protein are encoded by remaining ORFs [9]. Protein structural analyses and molecular modeling studies suggest that key drug-binding pockets in the viral enzymes are probably conserved across SARS-CoV2, SARS-CoV1, and MERS [10]. Accordingly, these tools can help to find anti-viral drugs against SARS-CoV2 with high specificity and lower side effects [11].

Via the S protein attachment to the host cells receptor, such as angiotensin-converting enzyme 2 (ACE2), SARSCoV2 may pass through the mucous membranes of the upper respiratory tract and enters the lungs. The most common symptoms which have been observed in several patients are fever and dry cough [11, 12]. Moreover, viral entrance to the peripheral blood from the lungs and viremia may occur. Then, the viral invasion could target organs that express ACE2, such as the lungs, gastrointestinal tract, heart, renal, and brain $[13,14]$.

The white blood cell (WBC) count in the early stage of the pathogenic process by SARS-CoV2 is generally normal or maybe slightly decreased [12], and most patients have lymphopenia [15]. Besides, the pro-inflammatory factors are significantly increased, which can aggravate the disease around 7-14 days after onset [16]. Most of the severely ill patients with SARS-CoV2 infection exhibit pneumonia (often called novel coronavirus infected pneumonia, NCIP) which may be associated with cytokine storm or Cytokines Release Syndrome (CRS): an imbalance between pro-inflammatory and anti-inflammatory factors. This life-threatening condition resulting from host immune dysfunction can lead to acute respiratory distress syndrome (ARDS) $[17,18]$. The massive infiltration of neutrophils, monocytes, and lymphocytes has occurred in this severe type of acute lung injury. Most patients who suffer ARDS have diffuse bilateral edema accompanied by reduced lung compliance, alveolar damage, and bronchoalveolar lumen hyaline deposition which result in hypoxic respiratory failure [19].

According to the Diagnosis and Treatment of Pneumonia Caused by SARS-CoV2 (updated to version 6), issued by the National Health Commission of the People's Republic of China, and due to the lack of specific anti-viral therapy against SARS-CoV2, current treatments have mainly focused on symptomatic and respiratory support [20].

Potential anti-COVID-19 therapy can be envisaged based on either direct effect on the coronavirus itself and/or on the immunotherapy options. One attractive approach is targeting receptor proteins on host cell surfaces that are often exploited by coronaviruses to bind via their receptor-binding domain 


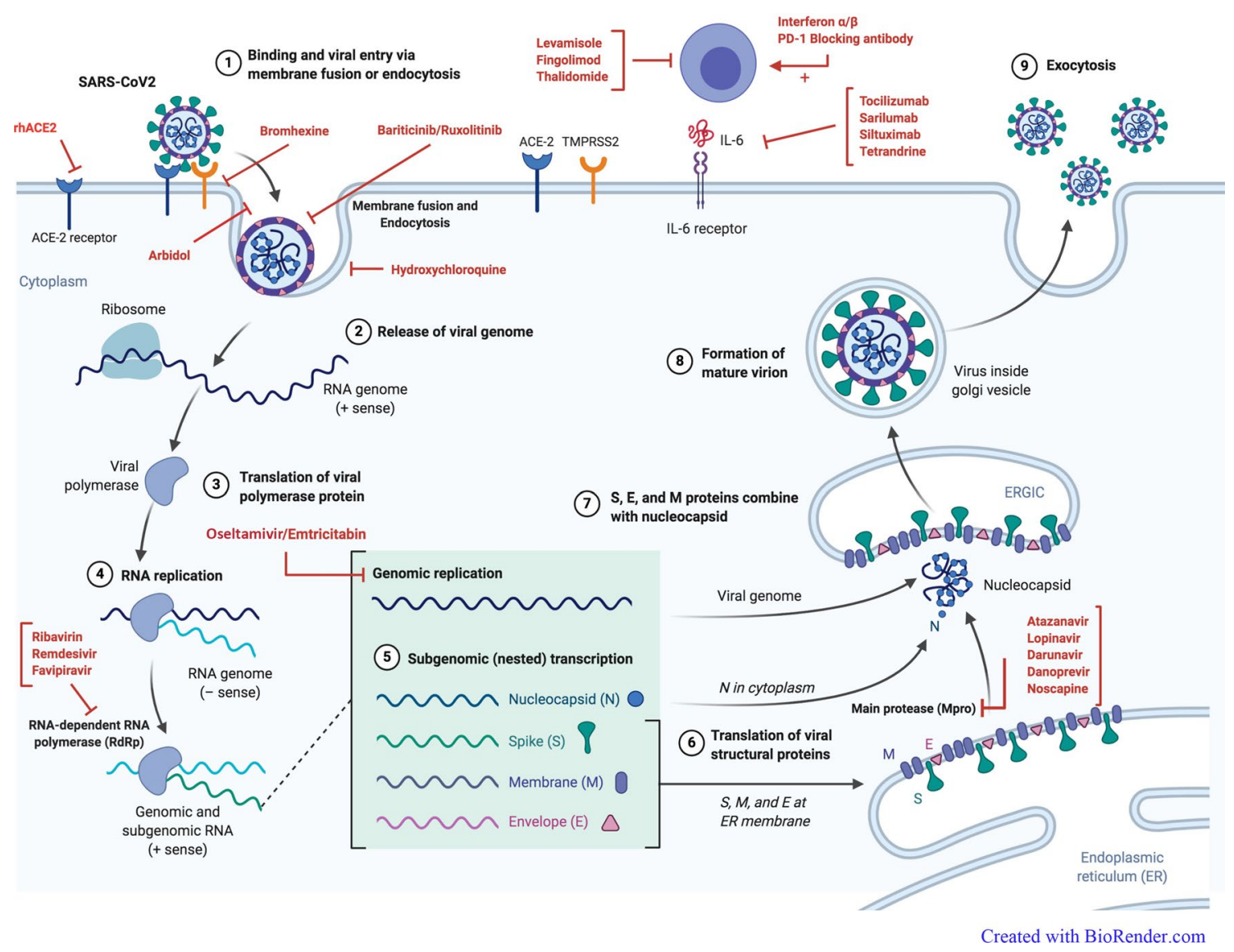

Fig. 1 The mechanism of actions of different drugs against COVID19. The figure illustrates the probable site of drugs effect during SARS-CoV2 infection including (1) binding and viral entry via membrane fusion or endocytosis (which are blocked by rhACE2, Bromhexine, Arbidol, Hydroxychloroquine, Ruxolitinib, and Baricitinib), (2) release of the viral genome, (3) translation of viral polymerase protein, (4) RNA replication, (5) genomic replication (which is inhibited by Oseltamivir and Emtricitabine), (6) translation of viral structural protein such as main proteases (which is inhibited by

(RBD). Blocking such virus binding to deny viral entry to human cells is, thus, an attractive therapeutic approach $[21,22]$. Besides, some therapeutic agents could effectively suppress viral replication by inhibiting the RNA-dependent RNA polymerase (RdRp). Considering the critical role of the innate immune response to coronavirus infection [23], blocking the signaling pathways involved in viral replication and self-assembly processes and production of virulence factors to restore the host's innate immunity are reasonable anti-viral approaches. Some related target proteins include RdRp, helicase, N-terminal RNA-binding domain (NRBD), C-terminal RNA-binding domain (CRBD), different nonstructural proteins such as Nsp3 (Nsp3b, Nsp3c, PLpro, and Nsp3e) and Nsp7-Nsp8 complex, 3CLpro, E-channel (E protein), Spike (S), and ACE2 [11].
Atazanavir, Lopinavir, Darunavir, Danoprevir, and Noscapine), (7) capsidation and RNA-dependent RNA polymerase (which is inhibited by Remdesivir, Favipiravir, and Ribavirin), (8) formation of the mature virion, (9) exocytosis. Other recommended drugs act on immune responses such as Tocilizumab and Siltuximab (via inhibition of IL-6), interferon-alpha, and beta (as the immunoenhancement agents), Fingolimod and Thalidomide (as the immunomodulating agents). The figure is "Created with BioRender.com"

Up to date, the intermediate host of SARS-CoV2 is still unknown, and effective prophylactics or therapeutics are not available to treat SARS-CoV2 infection. Some therapeutic agents are used off-label, alone or in combination, but we need further experimental data and validation to achieve a gold-standard therapeutic regimen with high efficacy and low side effect. In this review, the effects of different therapies on SARS-CoV2 with updated clinical trials (up to June 2020) are assessed. Different drugs against COVID-19 are classified according to the certain or probable mechanism of actions (Fig. 1), including inhibitors of viral replication, inhibitors of viral proteases, inhibitors of viral entry to the host cell, immunoenhancement, immunomodulating, immunosuppressive, anti-inflammatory, pulmonary vasoeffectors, and some anti-asthma/anti-tuberculosis drugs (Table 1). Overviews of several SARS-CoV2 clinical trials 


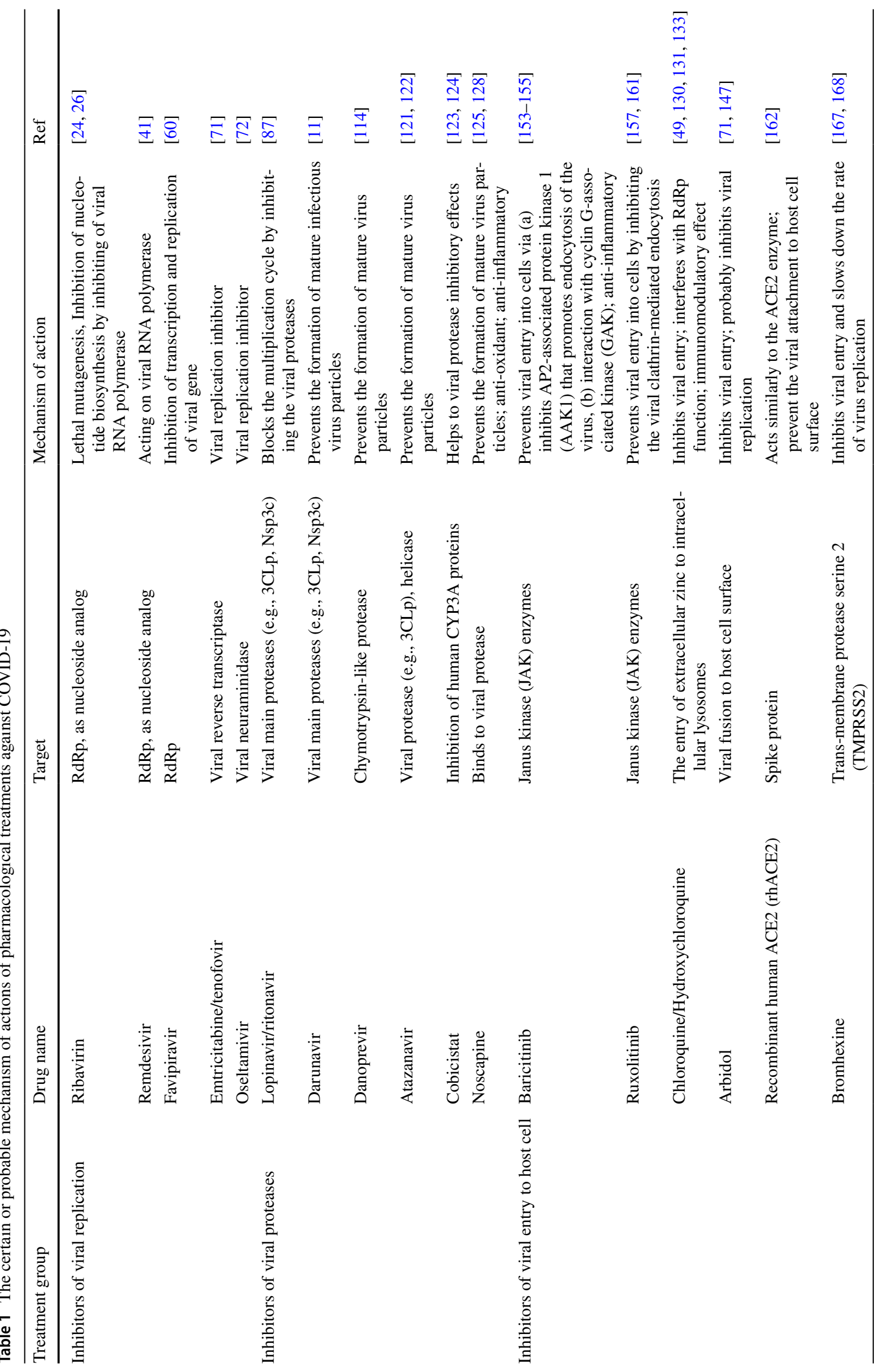




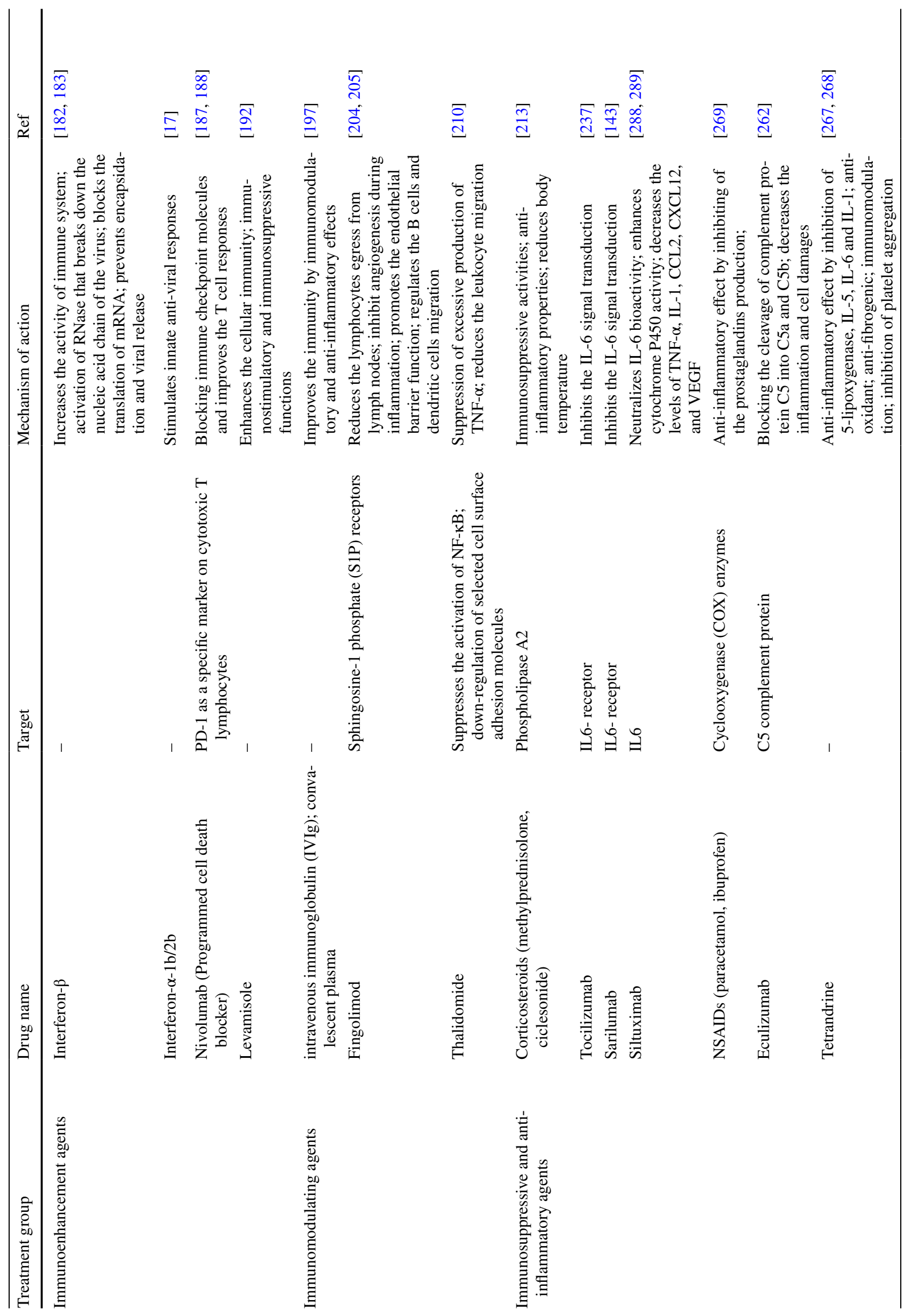


on previously approved drugs for the treatment of other viral infections that are currently underway are presented in the supplementary Table 2.

\section{Pharmacological treatments of COVID-19}

Inhibitors of viral replication

\section{Ribavirin}

The nucleoside analog, ribavirin (Virasol), is an anti-viral drug with broad-spectrum activity that is used for treating hepatitis $\mathrm{C}$, respiratory syncytial virus, and some viral hemorrhagic fevers (e.g., Lassa) [24, 25]. It had several mechanisms recognized by which ribavirin exerts its antiviral action, including lethal mutagenesis, chain termination as specific or nonspecific, and inhibition of nucleotide biosynthesis to target RNA viruses [26]. The desire-specific mechanism of ribavirin action has not been fully understood [27]. Also, it is a "broad-spectrum" drug and cannot kill coronaviruses in a targeted manner [11]. The proposed mechanism of action of ribavirin in SARS-CoV2 is through inhibition of synthesis of viral RNA and capping of mRNA [28]. The anti-viral activity of ribavirin against animal CoVs and SARS-CoV1 has been shown although its effectiveness along with interferon on MERS-CoV is contradictory. While several studies have shown the efficacy of ribavirin and interferon on their own [23, 29, 30], a combination of these drugs did not show positive outcomes in critically ill MERS patients [31, 32]. The efficacy of ribavirin mostly as combination therapy was assessed. The successful responses to ribavirin monotherapy and or combination therapy have been reported in several case report studies [33-37]. A multicenter, prospective, open-label, randomized, phase II trial was conducted in COVID-19 patients in Hong Kong. In the control group, the patients received lopinavir $400 \mathrm{mg}$ and ritonavir $100 \mathrm{mg}$ every $12 \mathrm{~h}$ for 14 days and the combination group received lopinavir $400 \mathrm{mg}$ and ritonavir $100 \mathrm{mg}$ every $12 \mathrm{~h}$, ribavirin $400 \mathrm{mg}$ every $12 \mathrm{~h}$, and three doses of 8 million international units of interferon $\beta$ - 1 b for 14 days. This combination therapy was well tolerated and reduced the time to negative nasopharyngeal swab and hospital stay in patients with mild to moderate COVID-19 [38]. Also, an open-labeled, single-center, prospective, randomized, and controlled clinical trial is performed to investigate the efficacy and safety of different anti-viral regimens (Ribavirin + Interferon $\alpha$-1b, lopinavir/ritonavir + IFN $\alpha-1 b$, and Ribavirin + lopinavir/ritonavir + IFN $\alpha-1 b)$ on 108 COVID19 patients. The results of this study can be helpful to provide obvious evidence for use of these regimens in the management of patients with mild to moderate COVID-19 [39]. 
It is also worth noting that ribavirin had several known adverse effects such as hemolytic anemia, hypocalcemia, and hypomagnesemia [40]. It is also contraindicated to autoimmune hepatitis, hemoglobin disorder, kidney failure, pregnant females or males with pregnant partners, and those with hypersensitivities to it [26]. Because of inconsistent benefit with ribavirin for COVID-19 and its substantial safety concerns, and very low quality of evidence, current data do not justify its use for the treatment of COVID-19. It seems that combination therapy is likely to provide the best chance for clinical efficacy. Therefore, extensive randomized controlled clinical trials are needed to confirm its efficacy on the mortality, virological and clinical outcomes of COVID-19. The effect of ribavirin in combination with other treatments is being evaluated in eight clinical trials (Table 2).

\section{Remdesivir}

Remdesivir (RDV), GS-5734, is a monophosphoramidate prodrug and adenosine nucleoside analog which is metabolized into active form GS-441524. The active form can reduce viral RNA production by acting on viral RNA polymerase [41]. RDV functioned at the stage of post-virus entry, which is in agreement with its putative anti-viral mechanism as a nucleotide analog [42]. It was developed by Gilead Sciences in 2017 for the treatment of Ebola virus infection but it is also used to treat Marburg virus, MERS, and SARS-CoV1 [41]. The RDV activity against many other human and zoonotic coronaviruses, including HCoV-NL63, HCoVOC43, HCoV-229E, mouse hepatitis beta coronavirus (MHV), bat beta coronaviruses HKU5, WIV1, and SHC014, and porcine delta coronavirus, was also observed [43-45]. Therefore, it can be introduced as a treatment choice for SARS-CoV2 [46].

The effect of RDV on SARS-CoV1 and MERS-CoV in human airway epithelial (HAE) cells was investigated and the reduced viral titers were observed, time-dependently [47]. Based on these observations, the expected role of GS-5734 in the SARS-CoV2 has been presented [48]. The obtained $\mathrm{EC}_{50}$ of RDV against SARS-CoV2 was $0.77 \mu \mathrm{mol} / \mathrm{L}$ using an in vitro study [49]. Based on molecular docking data, some viral proteins including RdRp, Nsp3b, E-channel, and TMPRSS2 were identified as potential targets for RDV [11]. RDV is currently undergoing a randomized, double-blind, controlled phase III clinical trial study in China. Moreover, for evaluating the efficacy and safety of RDV in patients with SARS-CoV2 pneumonia, two multicenter randomized, double-blind, placebo-controlled, Phase III studies were launched in February 2020 in China [50].

Two clinical trials of RDV have been implemented in China and this agent is the first investigational therapeutic which was approved by the FDA [42]. Despite the first reported case of SARS-CoV2 in the US used intravenous (IV) RDV on day 7 and achieved good results, the effectiveness of RDV is still questioned [51, 52]. Animal experiments indicated that both prophylactic and therapeutic RDV vs. lopinavir-ritonavir combination has effectively superior anti-viral effect in lung tissues, and lung function improvement [53]. Because of its promising results in SARS-CoV2 patients, RDV has now entered into phase III clinical trials [49].

According to the RDV activity against SARS-CoV2 in pre-clinical studies, a randomized and double-blind clinical trial study is ongoing to evaluate the RDV safety and effectiveness in 308 hospitalized patients with mild/moderate SARS-CoV2 pneumonia (NCT04252664) [54]. Another ongoing multicenter, double-blind, placebo-controlled, and phase III randomized clinical study has evaluated the RDV safety and efficacy in 453 hospitalized adult patients with severe SARS-CoV2 respiratory disease (NCT04257656) [55]. The details of the two trials are summarized in Table 2 [56].

By March 6, 2020, another Phase III randomized study was started to evaluate the RDV anti-viral activity and its safety in participants with severe SARS-CoV2. In this study, four arms including experimental and control groups are evaluated (NCT04315948) [57]. Another randomized, openlabel, phase III trial study was performed on 397 patients with severe COVID-19 who received $200 \mathrm{mg}$ of remdesivir on day 1 and $100 \mathrm{mg}$ once daily on subsequent days (NCT04292899) [58]. The results of this trial did not show a significant difference between a 5-day course and a 10-day course of remdesivir. Because of the lack of placebo control in this trial, it is not a test of the efficacy of remdesivir [59].

\section{Favipiravir}

Favipiravir (T-705) is an anti-viral drug that strongly inhibits RNA polymerases of RNA viruses. The mechanism of action is related to inhibition of transcription and replication of viral genes which eventually leads to the prevention of the viral RNA synthesis within infected cells [60]. Favipiravir has been shown to display anti-viral effects against many RNA viruses such as resistant influenza virus, arenaviruses, bunyaviruses, filoviruses, Rift Valley Fever, Yellow Fever, West Nile, Western equine encephalitis, foot-and-mouth disease virus, norovirus and avian influenza [61-65]. Because of its broad-spectrum anti-viral coverage, favipiravir was evaluated in the treatment of Ebola virus, Lassa virus, hantavirus, and now SARS-CoV2 [33, 66-68]. The efficacy of favipiravir versus LPV/r in SARS-CoV2 was evaluated in an open-label non-randomized control study in China. Changes in chest CT, viral clearance, and drug safety were surveyed in two groups of patients. The results showed a shorter viral clearance time, significant improvement in chest imaging, 
and fewer adverse effects in the favipiravir group compared to the control group [69]. In a randomized clinical trial, the efficacy and safety of favipiravir and arbidol were evaluated in 240 SARS-CoV2 patients. It was shown that the clinical recovery rate was higher in the favipiravir group. The time of fever reduction and cough relief in the favipiravir group was notably shorter than that in the arbidol group. But some anti-viral-associated adverse effects such as abnormal liver function tests, psychiatric symptom reactions, digestive tract reactions and raised serum uric acid were higher in the favipiravir group [70]. To evaluate the beneficial effects of favipiravir in coronavirus disease, several clinical trials on its efficacy are ongoing. The registered clinical trials have been reported and shown in Table 2 .

\section{Emtricitabine/tenofovir}

Tenofovir is a DNA synthesis inhibitor and approved as a human immunodeficiency virus (HIV) reverse transcriptase inhibitor. It has been suggested that tenofovir could be a potential drug candidate for SARS-CoV2 pulmonary disease, although the clinical effectiveness of this drug has not yet been evidenced for SARS-CoV2 [71]. At the time of preparing this manuscript, there was only one clinical trial that was designed to investigate the effectiveness of emtricitabine (FTC)/tenofovir alafenamide fumarate tablets (TAF) in combination with lopinavir/ritonavir. However, there are yet no data on treatment with emtricitabine/tenofovir in patients with COVID-19. This clinical study is summarized in Table 2.

\section{Oseltamivir}

Oseltamivir (Tamiflu) is an anti-viral neuraminidase inhibitor drug used for the treatment and prophylaxis of influenza $\mathrm{A}$ and B. Oseltamivir exerts its anti-viral activity by inhibition of the viral neuraminidase activity and viral replication. Oseltamivir seemed to suppress the virus reproduction in at least some cases [49, 72].

Coronaviruses are not known to utilize neuraminidase in viral replication; therefore, oseltamivir is unlikely to be of any therapeutic value. It was removed from the SARSCoV2 treatment protocol. The only study evaluating the effect of oseltamivir against coronaviruses showed it to be ineffective in preventing SARS-CoV1, even at high concentrations of the drug [73]. A retrospective, single-center case series of 138 hospitalized patients with SARS-CoV2 pneumonia in Wuhan, China showed that in about $90 \%$ of patients who received oseltamivir, no effective outcome was reported [15]. A phase IV clinical trial of anti-viral drugs for SARS-CoV2 pneumonia is underway in Wuhan China (NCT04255017) [74]. In this study, the efficacy of three antiviral drugs (arbidol hydrochloride, oseltamivir, lopinavir/ ritonavir) is being evaluated and compared (Table 2). In a retrospective case series of 99 patients with SARS-CoV2 at a single center in Wuhan, $76 \%$ of patients received anti-viral treatment, including oseltamivir. At the time of evaluation, $58 \%$ of patients remained hospitalized, $31 \%$ had been discharged, and $11 \%$ had died [75].

\section{Inhibitors of viral proteases}

\section{Lopinavir/ritonavir (LPV/r)}

Lopinavir/ritonavir (Kaletra $\AA$ ), as the main protease inhibitor, is mainly used for treating HIV infection [76]. Lopinavir/ ritonavir (LPV/r) is a combination of lopinavir and ribavirin and metabolized by the P450 cytochromes system in the liver. Because of the similarity in anti-viral activity between $\mathrm{LPV} / \mathrm{r}$ and LPV alone, the effect of LPV/r is mainly driven by LPV $[53,77]$. Pharmacokinetically, the CYP3A enzyme uses lopinavir as a substrate, but it is inhibited strongly by ritonavir (RTV). Since the enzyme activity of CYP3A can be affected by this drug, LPV/r should be cautiously used in combination with drugs that are metabolized by this enzyme [76]. Indeed, after inhibition of CYP3A by ritonavir, the serum concentration of LPV is increased [78].

SARS-CoV2 being an RNA virus like HIV [79], and also based on the clinical experiences in treating SARS-CoV1 [80] and MERS [81], lopinavir/ritonavir was first proposed for managing SARS-CoV2 infection [76]. Using the molecular weight calculation tool, the molecular mass of protease can be calculated from the functional motif showing protease function within the sequence of the virus [82]. On this basis, the molecular mass of the relevant proteases for the HIV and SARS-CoV2 differs from each other and is $21.6 \mathrm{kDa}$ and $33.8 \mathrm{kDa}$, respectively. By standard comparison techniques [83], the required dosage of LPV/r for SARS-CoV2 infection was about 1.56-times higher than that for HIV infection. Hence, based on the modeling study, if lopinavir/ritonavir is used for the management of SARS-CoV2, a double dosage of that used for HIV infection is suggested [79]. Also, the ongoing clinical trials are all designed to evaluate the efficacy of standard dosages of LPV/r in SARS-CoV2 patients.

The mechanism of LPV/r for treating coronavirus diseases is still unclear. Since coronaviruses, including SARS$\mathrm{CoV} 2$, synthesize polyproteins followed by hydrolysis to maintain their structural and functional proteins [9, 84-86], it is suggested that LPV/r may block their multiplication cycle by inhibiting their proteases [87]. It is initially hypothesized that lopinavir and ritonavir can inhibit the 3-chymotrypsin-like protease of SARS-CoV1 and MERS and may be associated with improvement of clinical outcomes in patients with COVID-19 in a non-randomized open-label trial [7]. However, more investigations are needed to find the inhibitory mechanism of HIV protease inhibitors on the 
3-chymotrypsin-like and papain-like proteases of SARSCoV2 [10].

Molecular docking results showed that non-structural protein-3c (Nsp3c) or E-channel can serve as possible targets of ritonavir. Additionally, lopinavir's possible targets include Nsp3b, helicase, and N-terminal receptor-binding domain (NRBD) [11]. It has also been shown that lopinavir and ritonavir produce strong interaction with the active site of SARS-CoV2 main protease (Mpro), in silico [88, 89].

Based on the prior research which has shown decreased mortality rates in patients with SARS-CoV1 infection by interferon-alpha (IFN- $\alpha$ ) and lopinavir/ritonavir, the use of these medications is recommended by National Health Commission of the People's Republic of China [90]. Indeed, the earliest protease inhibitor combination that was introduced for the treatment of SARS-CoV2 was lopinavir/ritonavir. Because of its notably lower incidence of adverse outcomes in comparison with ribavirin alone, the efficacy of lopinavir/ ritonavir was documented in several studies. Moreover, the lower incidence of ARDS, nosocomial infection, and death were observed in combination therapy with ribavirin [91]. Preliminary reports have shown that patients infected with SARS-CoV2 and treated with lopinavir could achieve viral clearance [92]. Altogether, these data support the assumption that some protease inhibitors may have an anti-viral effect by blocking the coronavirus main protease. Thus, based on its safety profile, LPV/r could be used as therapy and/or pre-exposure prophylaxis to reduce new infections by SARS-CoV2 [88].

Different clinical trials (for example, ChiCTR2000029539) have been initiated to test protease inhibitors such as lopinavir/ritonavir in patients with SARS-CoV2 [10]. LPV/r was associated with the highest total number of planned inclusions and was tested in several clinical trials. Furthermore, the use of this agent for the treatment of SARS-CoV2 has been described in case reports $[89,92]$ and a case series of patients infected with SARS$\mathrm{CoV} 2$ in Singapore [93]. Moreover, treatment by lopinavir/ ritonavir could significantly decrease the SARS-CoV2 viral loads in a Korean patient [92]. For investigation on LPV/r in patients with SARS-CoV2 infection, 9 clinical trials have been registered in China up to March 28, 2020 [78]. Several trials involve lopinavir-ritonavir treatment (Table 2), as the standard care arm, in comparison with the use of other drugs for SARS-CoV2 such as arbidol, danoprevir-ritonavir, and interferon inhalation [94]. Nevertheless, a recent clinical trial study on 199 patients with SARS-CoV2 infection showed that the treatment by lopinavir-ritonavir was not associated with clinical improvement in comparison with standard care procedures [95]. It appears that there have been some limitations in this study. First, it was not a blinded study; second, the delayed start of the treatment may attribute to the lack of effectiveness of LPV/r. Third, all patients enrolled in the study had an oxygen saturation $(\mathrm{SaO} 2)$ of $94 \%$ or less while they were breathing ambient air or a ratio of the partial pressure of oxygen $(\mathrm{PaO} 2)$ to the fraction of inspired oxygen ( $\mathrm{FiO} 2)$ of less than $300 \mathrm{mmHg}$. Hence, future studies are needed to verify the efficacy of LPV/r anti-viral treatment. The wide availability and manufacturing of lopinavir-ritonavir imply that it could be prescribed immediately for COVID-19. Several case reports and case series have used for this agent against SARS-CoV2. But, the trial results so far were disappointing [96]. Based on the direct inhibitory activity against viral replication, the detection of viral nucleic acid in patients has shown the inability of LPV/t to suppress the viral load. Thus, the whole effects of LPV/r were not simply observed, although some effects of the drug might be possible [96]. A clinical trial study on comparing favipiravir with lopinavir/ritonavir for the treatment of SARS-CoV2 has shown that this drug had a more potent anti-viral activity with little adverse effects than that of lopinavir/ritonavir [50, 97].

There might be some explanations for the unexpected or lower effects of lopinavir-ritonavir. The patient selection in late phases of COVID-19 infection and also significant tissue damages (with considerable mortality in the control group) could be the main reasons for the inability of lopinavir-ritonavir in whole treatments. In some cases, there is probable drug interference when LPV/r is used with other agents. Moreover, the drug concentration in the tissues where SARS-CoV2 is replicating is not detected and lopinavir could not have particular activity against SARS-CoV2. Inhibition of viral replication is needed by the relatively higher concentration of lopinavir-ritonavir as compared with the serum levels found in patients treated with this drug $[53,96]$.

Nevertheless to the efficacy of LPV which is indicated in most of the data, the adverse reactions should also be considered [78]. Diarrhea, vomiting, and rash are the most common adverse reactions of LPV/r [98]. The most frequently reported adverse reactions in patients who received LPV were diarrhea, nausea, and asthenia [99]. The changes in laboratory findings included high levels of total bilirubin, triglyceride, and hepatic enzyme activities have also been reported [100,101].

\section{Darunavir}

Darunavir (DRV), as the HIV protease inhibitor, can prevent the formation of mature infectious virus particles by the selective inhibition of the cleavage of Gag-Pol polyprotein in virally infected cells [11].

In February 2020, the inhibitory effect of DRV on SARSCoV-2 infection was announced by Chinese researchers. Cell experiments indicated that the viral replication was significantly inhibited by DRV at a concentration of $300 \mu \mathrm{M}[50$, 102]. It has been shown that darunavir in combination with 
Cobicistat (DRV/c) can significantly inhibit the replication of the SARS-CoV2 [91, 103]. This combination therapy was approved by the United States Food and Drug Administration (FDA) for the treatment of patients with AIDS. To enhance the pharmacokinetics and pharmacodynamics of darunavir, cobicistat, like ritonavir in LPV/r, can affect as a booster and inhibits the cytochrome P450 (CYP3A) [86, 104]. In addition to in vitro and clinical trial studies, several in silico studies have also confirmed the effectiveness of the anti-viral activity of DRV against SARS-CoV2. The potential therapeutic effect of DRV against SARS-CoV2 may be mainly due to its inhibitory effect on papain-like viral protease (PLVP) and Main protease (Mpro) [11, 105-107]. DRV has been shown to display a high ligand affinity and a potential candidate, capable of disrupting communication between SARS-CoV2 receptor-binding domain and ACE2. Hence, DRV is now suggested to be repurposed for the treatment of SARS-CoV2 infection for its possible disruption of cellular recognition, binding, and invasion. This drug has promising effects for an alteration of the viral tropism and the dynamics of human-to-human transmission when tested in a laboratory or clinical setting [108]. Clinical studies on the treatment of SARS-CoV2 by DRV are summarized in Table 2.

\section{Danoprevir}

Danoprevir is an investigational NS3/4A HCV protease inhibitor that had favorable effects against diverse $\mathrm{HCV}$ genotypes $[109,110]$. Since the non-structural proteins, such as proteases, are essential for the pathogenicity of coronaviruses, they serve as a good target for drug therapy [111]. It is proposed that the chymotrypsin-like protease of SARS$\mathrm{CoV} 2$ is structurally similar to other RNA viruses such as HCV $[112,113]$. On the other hand, in a molecular docking simulation study, simeprevir has a stronger interaction with connecting receptors than lopinavir/ritonavir, which is commonly used in different SARS-CoV2 treatment regimens [114]. The anti-viral mechanism of simeprevir and danoprevir is similar [115]. Recently, danoprevir was approved in China for the treatment of treatment-naive patients with non-cirrhotic genotype $1 \mathrm{~b}$ chronic hepatitis $\mathrm{C}$. It has also shown potent and broad-spectrum anti-viral effects on hepatitis C [116]. Moreover, in another molecular docking study, danoprevir has shown a potent inhibitory effect on SARS-CoV2 [107]. Therefore, it could be claimed that danoprevir can be a potential treatment in COVID-19. The efficacy of danoprevir has been confirmed for the treatment of hepatitis $\mathrm{C}$ and patients receiving this medication have been tolerated well [117]. There is a completed clinical trial (NCT04291729), which evaluated the efficacy of danoprevir combined with ritonavir in the treatment of COVID-19. The primary data reported from this clinical study demonstrated that all included patients had improved clinical and paraclinical parameters. Fortunately, all of the included patients in this study were discharged from the hospital [118]. However, this study is an open-label clinical trial with low sample size, and another clinical trial with a larger sample size is ongoing. While we can conclude that this treatment has some efficacy for the treatment of COVID-19 patients, further randomized clinical trials are needed to confirm the result of the aforementioned study. The characteristics of this clinical trial have been reported in detail in Table 2 .

\section{Atazanavir}

Atazanavir (ATV), with a mechanism of action of protease inhibition, is approved for treating HIV or AIDS [119]. As indicated in the preceding sections, non-structural proteins like protease, a critical enzyme for polyprotein processing in CoVs [120], are necessary for the pathogenicity of CoVs [111]. Hence, atazanavir prevents the formation of a mature virus particle and inhibits SARS-CoV2 infection. In a study based on uncovering SARS-CoV2 helicase inhibitors through molecular docking analysis, Borgio and coworkers showed that atazanavir may interrupt the activity of SARS-CoV-2 helicase [121]. The recent study by Beck and his co-workers's based on molecule transformer-drug target interaction (MT-DTI) also showed that atazanavir was the best among the tested compounds for inhibitory SARS-CoV2 3C-like proteinase activity and the observed rankings was in the order of atazanavir $>$ remdesivir $>$ efavirens $>$ ritonavir $>$ dolutegravir [122]. A clinical trial (IRCT20100228003449N30) is also in the process of evaluating the efficacy and safety of hydroxychloroquine plus lopinavir or atazanavir/ritonavir combination in patients with COVID-19 (Table 2).

\section{Cobicistat}

Cobicistat is used in the treatment of HIV/AIDS and its main mechanism is via inhibition of human CYP3A proteins with also some limited effect on CYP2D6 [123]. Through inhibition of the main protease, cobicistat is suggested to have potential in SARS-CoV2 therapy [124]. Since it is as effective as lopinavir/ritonavir for the treatment of HIV/AIDS and better tolerated due to its lower adverse effects (diarrhea $2 \%$ vs $27 \%$ ), its effects in patients with SARS-CoV2 are being assessed under phase III clinical trial (NCT04304053). Currently, another clinical trial is in the process that investigates the effects of cobicistat in the treatment of pneumonia associated with SARS-CoV2 (NCT04252274). The virological clearance rate of throat swabs, sputum, or lower respiratory tract secretions is being measured at day 7 of the treatment (Table 2). 


\section{Noscapine}

Terpenoids have numerous medicinal activities including anti-oxidant, anti-cancer, anti-bacterial, anti-inflammatory, and anti-viral effects through inhibition of virus proteases [125]. Noscapine (NOS) is a natural phthalideisoquinoline alkaloid that is extracted from the opium poppy, Papaver somniferum of the Papaveraceae family. It has been used for many years as a safe antitussive by suppressing the central nervous system $[126,127]$. Kumar et al. have shown that the noscapine derivative (23B) could bind to the protease of SARS-COV2 effectively at $300 \mathrm{~K}$ and inhibit protease function based on computer-drug design studies through docking and molecular dynamic simulations. Furthermore, they showed $325 \mathrm{~K}$ was the best temperature for binding of the noscapine derivative with protease [128]. Currently, a clinical trial (IRCT20160625028622N1) is in the process that investigates the effects of noscapine on clinical and pulmonary manifestations of COVID-19 patients (Table 2).

\section{Inhibitors of viral entry to the host cell}

\section{Chloroquine/hydroxychloroquine}

The sulfate and phosphate salts of chloroquine (CQ) are categorized as anti-malarial drugs. Hydroxychloroquine (HCQ) is also used both as an anti-malarial drug and for autoimmune diseases such as rheumatoid arthritis and systemic lupus erythematosus [129]. The proposed mechanism of anti-viral activity of CQ in SARS-CoV2 is via targeting endosomal low $\mathrm{pH}$ (acidification) needed for virus/ cell fusion, and interference with glycosylation of cellular receptors. Time-of-addition assay of CQ in Vero E6 cells showed anti-viral function at both entrances and post-entry steps of the infection with SARS-CoV2. The study by Wang et al. revealed that the $50 \%$ effective concentration $\left(\mathrm{EC}_{50}\right)$ of CQ was $1.13 \mu \mathrm{M}$ and the $\mathrm{EC}_{90}$ was $6.90 \mu \mathrm{M}$ which represents desirable viral inhibition at micromolar concentrations. In addition to direct anti-viral activity, CQ/HCQ specifically targets the entry of extracellular zinc to intracellular lysosomes where it interferes with RNA-dependent RNA polymerase function and coronavirus replication [130]. Furthermore, the immunomodulatory effect of CQ increases its anti-viral activity in vivo [49], although the exact mechanism of action of CQ needs further studies. The molecular mechanism of HCQ is similar to CQ and it can inhibit the cytokine storm via suppressing $\mathrm{T}$ cell activation [131]. In vitro, HCQ was shown to be more potent than CQ against SARS-CoV2 [132]. CQ and HCQ have been shown to inhibit SARS-CoV-2 replication [133]. A study by Gao et al. involving more than 100 patients reported that CQ phosphate is more effective than the control treatment group, who were treated using standard treatment without $C Q$, in preventing the pneumonia exacerbation, improving lung function in computed tomography (CT) images, accelerating a viral seroconversion, and reducing the disease period with no serious side effects. Hence, this drug is recommended for inclusion in the next version of the Guidelines for the Prevention, Diagnosis, and Treatment of Pneumonia Caused by SARS-CoV2 issued by the National Health Commission of the People's Republic of China [134]. Chinese experts recommend $500 \mathrm{mg}$ CQ phosphate twice daily for 10 days in patients with mild, moderate, and severe COVID19 pneumonia without contraindications to CQ [135]. One parallel, double-masked, randomized, phase IIb clinical trial suggests that the higher CQ dose regimen should not be recommended for critically ill COVID-19 patients because of its safety profile, especially when used concurrently with azithromycin and oseltamivir. This trial suffered from several limitations such as small sample size, absence of a placebo control group, lack of exclusion criteria regarding the QTc interval (defined as the interval between Q wave and T wave in electrocardiogram) at baseline [136]. CQ is considered for SARS-CoV2 on a prophylaxis basis because of its potential efficacy, safety profile, convenient availability, and low cost. Two prophylactic programs were suggested for CQ against SARS-CoV2: (1) CQ $8 \mathrm{mg} / \mathrm{kg} /$ day for 3 days in postexposure, in asymptomatic cases must be taken during hours after well-known exposure, (2) for chronic prophylaxis in the outbreak or endemic areas, CQ $500 \mathrm{mg}$ daily for 30 days and thereafter decreasing to $250 \mathrm{mg}$ daily for as long as the threat of infection is diminished [137]. But to date, there is a lack of evidence to support the efficacy of CQ or HCQ in preventing COVID-19 [138].

Different trial studies have shown that the use of HCQ in COVID-19 patients can improve clinical symptoms and patient survival [139, 140]. However, a study consisting of 181 patients with COVID-19 pneumonia (84 receiving HCQ within $48 \mathrm{~h}$ of admission and 97 that did not) reported no difference in Intensive Care Unit (ICU) transfer or mortality within 7 days and did not support the use of HCQ in patients with COVID-19 pneumonia [141].

The rapid fall of nasopharyngeal viral load in COVID19 patients has been reported after they received HCQ and azithromycin combination therapy [142-144].

The most common side effects of HCQ and CQ are gastrointestinal effects such as vomiting and diarrhea [131]. Neurologic side effects (including muscular weakness, diplopia, dyskinesia, seizures, myasthenic syndrome) and psychiatric side effects (including sleeplessness, agitation, psychosis, depression, anxiety, and confusion) have also been shown [145]. In long-term use, these drugs cause severe adverse effects including retinopathy and cardiomyopathy [131].

HCQ is generally less toxic, more soluble than CQ, and has lower tissue accumulation and adverse events than CQ. Also, HCQ can be recommended for pregnant women and is 
cheaper and more readily available in many countries [131]. Furthermore, HCQ is preferred because of no interference with other anti-viral drugs such as oseltamivir, lopinavir/ ritonavir, ribavirin, interferons, and immunoglobulins [133]. On the other hand, COVID-19 patients are more vulnerable to side effects of $\mathrm{CQ} / \mathrm{HCQ}$ because of their advanced age, co-morbidities including diabetes, obesity and cardiovascular disorder, and co-medication [145].

Because of the initial beneficial effect of CQ and HCQ in coronavirus diseases, several clinical trials on the efficacy of these drugs are currently ongoing. The officially registered clinical trials on these drugs are presented in Table 2. Of these, ten trials investigate their prophylactic effect against SARS-CoV2 in a healthcare setting or close contacts after exposure (Table 2). Because of insufficient clinical data and contradictory results, further well-designed clinical trials must be conducted for the suggestions of these drugs for the prevention or treatment of COVID-19 patients. Before using these drugs, the patients must also be having QTc. Also, long monitoring of side effects even after discontinuation of treatment due to prolong the half-life of CQ and HCQ must be performed. So, their off-label use outside of these trials is not recently recommended.

\section{Arbidol}

Arbidol (umifenovir), a small-molecular weight indolebased molecule, has a broad-spectrum anti-viral activity against numerous DNA and RNA viruses. It has been shown that arbidol can inhibit influenza A and B and hepatitis C viruses entry into host cells by blocking viral fusion [146, 147]. For prophylaxis and treatment of upper respiratory tract infections caused by influenza A and B viruses, arbidol has been approved in Russia and China [148]. Other studies also showed that arbidol hydrochloride can inhibit the fusion of the virus membrane to the host cells and block the virus replication [11]. In recent years, the effectiveness of arbidol against both SARS-CoV1 and MERS-CoV has been shown in many studies [11]. Due to a lack of significant adverse effects, arbidol is patented for SARS-CoV1 treatment [147]. Since the anti-viral effects of arbidol against SARS-CoV1 have been confirmed in cell-based experiments [149], it has been suggested to be a drug of choice for SARS-CoV2 treatment [17]. An in vitro study showed that arbidol can effectively inhibit SARS-CoV2 replication [71]. The dosage regimen of arbidol in adults with SARS$\mathrm{CoV} 2$ was recommended to be $0.2 \mathrm{~g}$ any time, twice daily, not to be administered over 10 days [150]. So far, the dosage regimen of arbidol in children with SARS-CoV2 has not been recommended [76]. It has been shown that arbidol may have effective inhibitory function against SARS-CoV2 infection at the concentration of $10-30 \mu \mathrm{mol} / \mathrm{L}$, and can also reduce the virus's pathological effects in host cells $[50,102]$.
Molecular modeling studies have shown that some proteins of SARS-CoV2 such as spike, E-channel, Nsp7-Nsp8 complex, Nsp14, and Nsp15 may interact with arbidol [11].

Several ongoing clinical trials are designed to evaluate the anti-viral efficiency of arbidol against SARS-CoV2 (Table 2). Moreover, this drug has been used as arbidol hydrochloride with oseltamivir and LPVr, and also in comparison with lopinavir-ritonavir, and interferon- $\alpha-2 a$. Moreover, the arbidol and lopinavir/ritonavir (LPV/r) combination treatment has been compared with LPV/r alone in a retrospective cohort study on patients with SARS-CoV2. It was found that the desired clinical response in the arbidol-LPV/r combination group was better than LPV/r alone [151]. A randomized controlled study on treatment of SARS-CoV2 by arbidol and Kaletra has also shown that arbidol could significantly reduce the incidence of severe SARS-CoV2 and had a better therapeutic effect [17].

There are some adverse reactions for arbidol therapy such as nausea, diarrhea, dizziness, and elevated serum aminotransferase. Moreover, arbidol should be administered with caution in patients with liver dysfunction because of its metabolism by the liver. Arbidol also can compete with high binding rate drugs to plasma proteins and increase the concentration of combined drugs, due to the $89.2-91.6 \%$ of protein-binding rate [76].

\section{Baricitinib}

Baricitinib is an anti-rheumatic medication for the treatment of refractory rheumatoid arthritis [152]. The primary anti-inflammatory mechanism of baricitinib in rheumatoid arthritis is through inhibition of Janus kinase (JAK) enzymes [153]. In SARS-CoV2, however, baricitinib prevents viral entry into cells through different mechanisms. It inhibits AP2-associated protein kinase 1 (AAK1): an enzyme that promotes endocytosis of the virus. Additionally, baricitinib interferes with viral endocytosis through interaction with cyclin G-associated kinase (GAK). Baricitinib is also suggested to reduce inflammation through inhibition of JAK1/2 enzymes [154]. Accordingly, baricitinib may have positive clinical effects in COVID-19 patients and could be an alternative option for the treatment of COVID-19, especially in patients with concurrent rheumatoid arthritis disorder. However, the inhibition of JAK-STAT kinase by baricitinib interferes with the anti-viral activity of innate interferons [155]. Furthermore, baricitinib can produce some symptoms of upper respiratory tract infections, nausea, and thrombosis in rheumatoid arthritis patients receiving this medication. Therefore, the efficacy and safety of baricitinib in patients with COVID-19 infection is not yet apparent. At the time of writing this article, there are several registered clinical trials and observational studies on the efficacy and safety of baricitinib for the treatment of COVID-19. One of them is 
completed and the primary outcome in this pilot study was the evaluation of baricitinib's safety. It did not increase the risk of infections, cardiovascular, and hematologic adverse effects after 2 weeks of treatment. On the other hand, treatment with baricitinib was associated with better clinical response and respiratory functions after 2 weeks of treatment when compared with a baseline assessment [156]. Nevertheless, this study is an open-label, non-randomized clinical trial. To make a clinical decision on the efficacy and safety of this drug, we need further studies with larger sample sizes. The characteristics of registered clinical trials have been reported in detail in Table 2 .

\section{Ruxolitinib}

JAK enzymes mediate signals from the cell surface receptors for various cytokines and growth factors that have a key role in inflammatory function. Ruxolitinib is a small molecule inhibitor of JAKs including JAK1 and JAK2 [157]. Among JAK inhibitors, ruxolitinib is currently used in patients who suffer myelofibrosis. In this hematological condition, ruxolitinib can rapidly improve the patient's quality of life by reducing the secretion of pro-inflammatory cytokines, such as IL- 1, TNF- $\alpha$, IFN- $\gamma$, and IL-6 [158-160].

Based on similar inhibitory function as baricitinib in blocking the viral clathrin-mediated endocytosis, Ruxolitinib might probably be useful in COVID-19. Recently, Stebbing and colleagues reported the possibility of employing JAK1/2 inhibitors in COVID-19, with more focus on baricitinib, ruxolitinib, and fedratinib, due to their powerful anti-inflammatory action, but with also warning about their possible negative effects in the infection control [161]. Moreover, an adaptive Phase II/III clinical trial was conducted, with a focus on the assessment of ruxolitinib's safety in the first 20 participants (Phase II), followed by a much broader assessment of efficacy, while continuing to monitor safety, in an additional 60 participants (Phase III, total participants across Phase II/III $n=80$ ). Both phases are singlearm, open-label, and occur at a single site at the University of Colorado Hospital. Study participants received $10 \mathrm{mg}$ twice daily of ruxolitinib for 14 days and followed for up to 29 days. Data from participants in this study would be compared with data from other COVID-19 patients not receiving ruxolitinib (NCT04348071). It seems that ruxolitinib might lower the hyper inflammation caused by the virus, which would prevent damage to the lungs (e.g., ARDS) and possibly other organs.

\section{Recombinant human ACE2}

Recombinant human angiotensin-converting enzyme 2 ACE2 (rhACE2) acts similarly to the ACE2 in the human body. It has the potential to treat ARDS for many reasons.
During ARDS, the activation of the ACE causes vasoconstriction in pulmonary vessels, causing a lung injury induced by hypoxia. Conversely, the activation of ACE2 has a likely protective effect against lung damage in ARDS. Therefore, rhACE2 could act as a potential therapy for ARDS in different situations [162]. ARDS and multiorgan dysfunction constitute significant complications in patients with COVID-19 infection. Therefore, the use of rhACE2 could be a practical approach in COVID-19 patients with severe symptoms [163]. Unfortunately, there is yet no completed study on the efficacy and safety of this intervention. There are only two ongoing clinical trials on the efficacy of rhACE2 in the treatment of patients with COVID-19. The characteristics of registered clinical trials are described in detail in Table 2.

\section{Bromhexine}

Bromhexine is categorized as a mucolytic agent and is primarily used for bronchopulmonary infections with unusual mucous secretions. Previous studies have shown that it has some efficacy in respiratory infections. Bromhexine causes a decrease in the severity of this infection by acting on mucus production, mucus clearance, and enhancement of anti-biotic penetration $[164,165]$. The host proteases play an important role in the pathogenicity of various viruses such as influenza viruses, Ebola viruses, and coronaviruses through different mechanisms [166]. In the case of coronaviruses, proteases probably serve as an activator of S-glycoprotein, which is a virusbinding protein [167]. The trans-membrane protease serine 2 (TMPRSS2) is a type of protease that is demonstrated to be pivotal for the pathogenicity of influenza viruses, SARS-CoV1, MERS, and other coronaviruses [167, 168]. Additionally, the structure of the target receptor of SARSCoV2 is approximately similar to SARS-CoV1 [169]. Accordingly, the TMPRSS2 is a probable target protease for SARS-CoV2 [167]. Therefore, inhibition of proteases, especially TMPRSS2, could slow down the rate of virus replication [170]. Bromhexine likely acts as an anti-viral drug through inhibition of TMPRSS2. However, there is no rationale in using bromhexine as monotherapy because of its adverse effects in high doses and lack of clinical evidence of its efficacy against SARS-CoV2 [170, 171]. Therefore, bromhexine could be an additional therapeutic option along with other anti-viral drugs. Up to now, there are three undergoing registered clinical trials on the safety and effectiveness of bromhexine in patients with suspected and mild COVID-19 infection. However, there is still no reported data on the efficacy of bromhexine in the treatment of COVID-19. The characteristics of the registered clinical trials have been reported in detail in Table 2 . 


\section{Immunoenhancement agents}

\section{Interferon-alpha $(a)-1 b / 2 b$}

Interferons (IFN) can participate in the control of coronavirus infection by enhancing the innate immune response [23]. Despite there is no evidence to validate the beneficial effects of passive immunization therapy, the use of interferon could theoretically be helpful for the treatment of viral infections [172]. To stimulate innate anti-viral responses in patients infected with SARS-CoV2, the administration of pegylated interferon- $\alpha-2 a$ and $2 b$, which were approved for the treatment of HBV and HCV, could be used. Despite the initiation of some clinical trial studies which used pegylated interferon plus an anti-viral nucleoside compound such as ribavirin, the synergistic activity against SARS-CoV2 is unclear. Evaluating subcutaneous interferon therapies should be closely monitored because of multiple adverse effects. Thus, it may be required to reduce or discontinue therapy [17].

It has been shown that three virulence factors including Nsp1, Nsp3c, and ORF7a assist the escape of coronavirus from the host immune system by interfering with innate immunity. Studies have shown that Nsp1 has a specific degradation activity on host mRNA by interacting with ribosomal small subunit and also can inhibit type-I interferon production $[173,174]$.

The effectiveness of synthetic recombinant IFN $\alpha$ in the treatment of SARS-CoV1 infected patients has been proven in clinical trials [175]. Therefore, the use of interferon in the treatment of SARS-CoV2 may have beneficial effects. Studies on evaluation of the effects of type-I interferon (IFN-I) on SARS-CoV1 infection were used as insights for recommending interferons for SARS-CoV2 infection. It is expected that SARS-CoV2 is sensitive to IFN-I pretreatment because of its similar viral replication kinetics to SARS-CoV1. Since IFN $\alpha-1 b$ and $2 b$ are subtypes of the IFN-I superfamily, the in vitro experimental effects of them might be shared by all other subtypes [176].

Different anti-virals such as interferon- $\alpha-1 b / 2 b$ have been approved in the latest version of the guidelines for the treatment of SARS-CoV2 issued by NHC of the People's Republic of China [177]. Patients with SARS-CoV2 are being recruited in randomized trials to evaluate the efficacy of different anti-virals plus interferon- $\alpha$. Some studies used interferon- $\alpha$ via aerosol inhalation for 2 weeks, while others used human recombinant interferon- $\alpha-1 b$ (Cerrokin) using nebulization. Moreover, the administration of recombinant interferon- $\alpha-1 b$ as an eye drop and nasal drop has been used in two studies (ChiCTR2000030480 and ChiCTR2000029989). The recombinant super compound interferon (rSIFN-co) has also been compared to interferon- $\alpha$ using nebulization (ChiCTR2000029638). Some studies claimed that ribavirin and interferon- $\alpha$ have a synergistic effect when applied at the early stage of SARS$\mathrm{CoV} 2$ infection. Health professionals were not fully satisfied yet, however, so further clinical research in this field is needed [31]. Evidence showed that the treatment using interferons, with or without ribavirin, and lopinavir/ritonavir are most likely to be beneficial against SARS-CoV2. The effectiveness of this treatment mainly depended on the timing of the treatment [91]. Moreover, some case reports used interferon therapy accompanied by other anti-virals for the treatment of patients with SARS-CoV2 [178-180].

\section{Interferon-beta ( $\beta$ )}

Interferon- $\beta$ is categorized as a type-I interferon and is the first and main drug approved for the treatment of relapsing-remitting MS (RRMS) patients and it has two classes: interferon $\beta$-1a and interferon $\beta$-1b [181]. Interferons can increase the immune system in multiple ways, via anti-viral, anti-proliferative, immunomodulatory, and developmental activities [182]. The mechanism of action of the anti-viral activity of interferon- $\beta$ is related to the activation of RNase that breaks down the nucleic acid chain of the virus. Also, it blocks the translation of RNA, prevents encapsidation, and viral release [183]. It seems that its administration at a later stage of COVID-19 might worsen the cytokine storm and exacerbate inflammation [184].

The synergistic inhibitory effect of ribavirin with interferon- $\beta$ on replication of SARS-associated coronavirus has also been demonstrated in animal and human cell lines [185]. In a retrospective study on 256 patients with COVID19 pneumonia, interferon- $\beta 1 \mathrm{~b}$ therapy was not associated with reducing in-hospital mortality. This study however suffered from several limitations including the retrospective design, selection and unmeasured confounding bias, and no evaluation of differences between the two groups in terms of virus elimination, and RT-PCR monitoring [183]. Furthermore, the effect of interferon- $\beta 1 \mathrm{~b}$ as a combination therapy (with lopinavir, ritonavir + ribavirin) was investigated in an open-label, randomized, phase II trial in COVID19 patients in Hong Kong. This combination therapy was safe and superior to lopinavir-ritonavir alone in reducing virus shedding, improving symptoms, and accelerating the discharge of patients with mild to moderate COVID-19. An important limitation of this study is open-label design, without a placebo group, and confounded by a subgroup deleting interferon- $\beta 1 \mathrm{~b}$ within the combination group, depending on time from symptom onset [38]. The most common side effect of interferon- $\beta$ is a flu-like syndrome (such as headache, fever, and arthralgia). Other side effects of it include injection site reactions, depression, liver enzymes elevation, and hematologic abnormalities [186]. In eight Iranian clinical trials, the efficacy of interferon- $\beta$ in COVID-19 in combination with conventional treatment is undergoing. 
Furthermore, eight clinical trials registered in ClinicalTrials.gov explore the effects of interferon- $\beta$ in COVID-19 (Table 2). The outcomes of these trials will give insight into the promise of interferon- $\beta$ for COVID- 19 therapy. The effect of interferon- $\beta$ in COVID-19 is unknown, but further phase III clinical trials with interferon $\beta-1 \mathrm{~b}$ as a basic treatment with a placebo control group should be considered.

\section{Programmed cell death 1 (PD-1) blocking antibody}

PD-1 blocking antibody is among a class of drugs that are commonly used for the immunotherapy of cancer. The mechanism of action is through blocking immune checkpoint molecules. Recently, a PD-1 blocking antibody named "Nivolumab" was approved for the treatment of many cancers $[187,188]$. On the other hand, the administration of the PD-1 blocking antibody in septic patients was associated with reversal of immune dysfunction due to infection, and it improved survival rate [189]. PD-1 is a specific marker on exhausted T cells during viral infections. As a result of the expression of PD-1 on cytotoxic T cells, the anti-viral activity of these cells diminishes in a variety of virus infections such as SARS-CoV2. Hence, blocking PD-1 with PD-1 blocking antibodies would reverse $\mathrm{T}$ cell exhaustion in these patients [190]. The most adverse effects of PD-1 blocking antibodies in other indications were fatigue, pruritus, and diarrhea. Fortunately, most of the treatment-related adverse effects were low grade and tolerable [191]. Recently, a new RCT (NCT04268537) in China on the efficacy of the PD-1 blocking antibody for the treatment of COVID-19 patients has been registered. The estimated participants in this RCT are 120 patients. To decide on the effect of this drug on the COVID-19, information from clinical studies is needed. The characteristics of a registered clinical trial are described in detail in Table 2.

\section{Levamisole}

Levamisole, a levisomer of tetramisole and belongs to the anti-helmintic class of medications and as the first member of the new class of drugs which enhances the cellular immunity, is a synthetic low-molecular weight compound. Based on dosage and timing in clinical administration, both immunosuppressive and immunostimulatory functions are observed for levamisole [192]. According to a previous study, a combination of levamisole and ascorbic acid in vitro can reverse the depressed helper/inducer subpopulation of lymphocytes in measles. The abnormality in lymphocytes by levamisole could be reproduced by treating normal lymphocytes with measles virus in vitro. Therefore, levamisole could also be considered for the treatment of COVID-19 $[24,193]$.
Common side effects of levamisole are mild and infrequent and include rash, gastrointestinal disorders (nausea, abdominal cramps, taste alteration), alopecia, arthralgia, flulike syndrome, and rarely agranulocytosis [194].

Heretofore, three randomized clinical trials are registered in Clinicaltrial.gov to evaluate the efficacy of the levamisole in the treatment of COVID-19. In the first double-blind, randomized, placebo-controlled phase II trial, the efficacy of oral levamisole and formoterol plus budesonide inhaler in combination with standard care was evaluated in the treatment of 30 patients with COVID-19 [195]. Two other Egyptian double-blind randomized placebo-controlled trials evaluate the efficacy of levamisole and isoprinosine in COVID-19 [195, 196].

\section{Immunomodulating agents}

\section{Intravenous immunoglobulin}

Over the past decades, the use of intravenous immunoglobulin (IVIg) could improve the immunity and prognosis of severe infections by the immunomodulatory and antiinflammatory effects. Moreover, the cytokine storm could be inhibited by the use of IVIg [197]. Hence, IVIg therapy may improve the prognosis of critically ill patients with SARS-CoV2. The efficacy of IVIg therapy on 80 patients with severe SARS-CoV2 pneumonia is ongoing [122].

In a series of treatments including ribavirin, lopinavir and ritonavir, corticosteroids, type-I interferon, IVIg, or convalescent plasma $(\mathrm{CP})$, the effects of these treatments on the rate of morbidity and mortality in SARS-CoV1 patients, ARDS development in adult patients, and SARS-CoV1 replication were investigated [198]. The immunomodulatory activity of convalescent plasma as well as IVIg therapy has been shown in studies [199]. This effect, as a treatment for SARS-CoV1 infection, has also reported in other Chinese studies [200, 201]. In a study based on the clinical and epidemiological investigations of ten children with SARSCoV2 infection who had nonspecific symptoms, all patients were not required respiratory support or ICU and received anti-viral therapy with IFN $\alpha$ at admission. Despite becoming negative in the nasopharyngeal swab specimen test, the viral nucleic acid in the rectal swab was positive, even with medication [202]. Therefore, the treatment could not completely reverse the SARS-CoV2 infection.

The convalescent plasma containing neutralizing antibody titers above 1:640 at one dose of $200 \mathrm{~mL}$ was transfused to ten patients with severe SARS-CoV2 infection who were under maximal supportive care and anti-viral agents. The primary outcome was the safety of CP transfusion. The improvement of clinical symptoms and laboratory parameters within 3 days after transfusion was also considered as the second outcome. The findings have shown that the 
level of neutralizing antibody increased rapidly up to 1:640 in five cases, while that of the other four cases maintained at a high level. Moreover, the clinical symptoms and the laboratory results, such as lymphocyte counts and C-reactive protein (CRP), were significantly improved. The viral load was undetectable after transfusion in seven patients who had previous viremia. Radiological findings showed varying degrees of absorption of lung lesions within 7 days. Due to the small sample size of this study, the clinical effectiveness and optimal dosage/and time point of CP transfusion are needed further investigation in larger populations [203].

\section{Fingolimod}

Fingolimod (FTY720) is an oral immunomodulatory drug that inhibits lymphocytes through sphingosine-1 phosphate (S1P) receptors [204]. Fingolimod is the first FDA approved oral drug for the treatment of relapsing-remitting multiple sclerosis (RRMS). S1P down-modulation slows the lymphocytes egress from lymph nodes and its recirculation can inhibit angiogenesis during inflammation. This drug can promote the endothelial barrier function and also regulates the B cells and dendritic cell migration [205]. The use of immune modulators along with ventilation support could prevent the development of ARDS in severe or critical patients with SARS-CoV2 according to some pathological findings of pulmonary edema and hyaline membrane formation [206].

Moreover, a prospective, non-randomized, no placebocontrolled, phase II clinical trial to study the efficacy of the fingolimod in the treatment of COVID-19 was started by February 2020 in Fuzhou, China [207].

\section{Thalidomide}

Thalidomide is an immunomodulatory drug with its immunologic effect varies under different conditions. Its effects can be related to the suppression of excessive TNF- $\alpha$ production and down-regulation of selected cell surface adhesion molecules involved in leukocyte migration. It has reported that the combination of thalidomide and celecoxib could improve severe pneumonia in SARS-CoV2 patients [208]. The drug suppresses the activation of NF- $\mathrm{\kappa B}$ that promotes the production of pro-inflammatory cytokines such as TNF- $\alpha$ and IL-8. It is thus suggested to benefit SARS-CoV2 pneumonia [209].

Chen et al. reported a case of SARS-CoV2 pneumonia successfully treated with thalidomide in combination with a low-dose glucocorticoid. Based on the results, they stated that thalidomide could be used to reduce oxygen consumption and relieve digestive symptoms in SARS-CoV2 patients. Also, thalidomide can be used as adjuvant therapy in COVID-19 patients [210].
Clinical trials of thalidomide treatment for SARS-CoV2 pneumonia are underway in China (NCT04273529 and NCT04273581). The first study (NCT04273529) is a prospective, multicenter, randomized, double-blind, placebo, parallel-controlled clinical study that evaluated the efficacy and safety of thalidomide in the adjuvant treatment of moderate COVID-19 pneumonia (Table 2). Another study has been designed to evaluate the safety and efficacy of thalidomide combined with low-dose hormone adjuvant therapy for severe SARS-CoV2 patients (Table 2).

\section{Immunosuppressive agents}

\section{Glucocorticoids}

Corticosteroids are classic examples of immunosuppressive drugs. With their ability to stop or delay the progression of pneumonia, they have been proved to be effective for the treatment of ARDS [211, 212]. In addition to immunosuppressive activities, corticosteroids display potent anti-inflammatory properties with implications to reduce systemic inflammation, suppress exudative fluid in the lung tissues, promote absorption of the inflammasome, and prevent further diffuse alveolar damage. Through these mechanisms, corticosteroids can relieve hypoxemia and effectively protect the lung by preventing further progression of respiratory insufficiency [213]. Furthermore, corticosteroids can reduce body temperature and help alleviate the poisoning symptom caused by hyperthermia [214]. During the SARS-CoV1 and MERS-CoV outbreak, it was shown that the administration of corticosteroids may be associated with some adverse outcomes such as delayed viral acid nucleic cease from the blood [199, 215-217]. Moreover, the association between systemic corticosteroid therapy with an increased mortality rate in these pulmonary diseases has been indicated [215, 218]. Whether glucocorticoid therapy is definitively beneficial in the clinical management of these two coronavirus infections is still being debated [216, 219-221].

Based on six studies on 574 patients with ARDS of any cause who received corticosteroid therapy, there is no sufficient evidence to recommend this treatment [222].

According to the WHO and CDC (Centers for Disease Control and Prevention) recommendations, the corticosteroids should not be routinely utilized in the treatment of SARS-CoV2 unless other indications such as exacerbation of chronic obstructive pulmonary disease or septic shock exist [223].

Chinese reports revealed that many patients during the outbreak of SARS-CoV2 have received corticosteroid therapy. However, the available recommendations for the routine administration of corticosteroids are not sufficient $[224,225]$. Because of the immediate clinical importance of harm or benefit from corticosteroids, further evidence 
for or against corticosteroid therapy will be provided by the outcomes of ongoing clinical trial studies [224, 226, 227].

In patients with SARS-CoV2 who failed to respond to low-dose corticosteroid therapy, short-term moderate-dose corticosteroid plus immunoglobulin administration was effective for reversing the continued deterioration [228]. It is worth noting that there are some limitations to this study. First, the very small sample size $(n=10)$ was considered. Second, the moderate dosage of methylprednisolone $(160 \mathrm{mg} /$ day $)$ is not recommended and the usage of steroids in pneumonia due to SARS-CoV2 is still controversial and being debated. The decreased risk of the mortality rate was reported in SARS-CoV2 patients who developed ARDS after treatment with methylprednisolone. So, the administration of methylprednisolone may be beneficial for patients who develop ARDS [229]. Moreover, it has been recently reported that dexamethasone could reduce 28-day mortality in hospitalized patients who received respiratory supports (NCT04381936) [230].

Early diagnosis and timely treatment are critical for SARS-CoV2 infection. It is necessary to protect important organs in infected patients with pneumonia and effective measures, such as corticosteroid treatment, should be considered to prevent the development of ARDS in severe patients [231]. In addition to the timing of treatment, it is also important to master the treatment duration and choose appropriate corticosteroid formulations and dosage. The basic principles of corticosteroid formulations selection lie in the following two aspects: a short half-life and strong penetrating ability. The corticosteroid formulation used in one study cohort for methylprednisolone was with a median effect half-life of 12-36 h, which has been proved to be associated with a better intensity of immunosuppression [232].

There are insufficient data on the effectiveness of adjunctive glucocorticoid therapy in SARS-CoV2-infected patients with severe lower respiratory tract infections [233]. Based on the available evidence, the proper use of low-dose corticosteroids may provide survival advantages for critically ill patients with SARS-CoV2. However, this treatment in patients with refractory ARDS, sepsis, or septic shock should be strictly performed according to the recommended guidelines. For clinical management of critically ill patients with SARS-CoV2, the low-dose and short-time corticosteroids therapy (methylprednisolone, $<1 \mathrm{mg} / \mathrm{kg}$ body weight, no more than 7 days), besides the adverse reaction monitoring, would be more beneficial. Moreover, due to the delayed adverse effects in these patients, it is essential to perform long-term follow-up (6 months-3 years) studies [234].

The systemic corticosteroid treatment along with antiviral agents including neuraminidase inhibitors (oseltamivir, peramivir, zanamivir, etc.), ganciclovir, acyclovir, and ribavirin, as well as methylprednisolone, which have recommended previously in clinical practice for the treatment of influenza virus $[15,24]$, are invalid for SARS-CoV2 and, thus, not recommended [8]. Corticosteroid therapy for the treatment of SARS-CoV2-induced lung injury or shock has not been recommended [224].

The systemic steroids should not be used for children with SARS-CoV2. Since there is not significant lung inflammation in children with SARS-CoV2, immunomodulation is not required. However, corticosteroid therapy may be useful when children require ventilation and develop ARDS [229]; although the beneficial effects of this treatment are not consistent and accurate [235].

An in vitro study has shown that ciclesonide, like an inhaled corticosteroid, can suppress SARS-CoV2 replication in cultured cells at low concentrations and the $\mathrm{EC}_{90}$ was $6.3 \mu \mathrm{M}$. Ciclesonide also exhibited low cytotoxicity and potently suppressed viral replication. Mutation analysis has shown that non-structural protein-15 (NSP15) can be resistant to ciclesonide. This observation suggests that the effect of ciclesonide was specific to coronavirus, and it may, thus, be a candidate drug for the treatment of SARS-CoV2 infection. In comparison with ciclesonide, other corticosteroids including cortisone and prednisolone, which are commonly used for systemic steroid treatment, have been tested against SARS-CoV2. Viral replication was not suppressed by dexamethasone and fluticasone [236]. High concentrations of ciclesonide can be administered to infants, because of its safety. Since ciclesonide does not significantly enter the bloodstream and remains in the lung tissue, ciclesonide inhalation can reduce viral replication and host inflammation in the lungs. Hence, the evaluation of ciclesonide with consideration of the balance between its benefit and risk is recommended for the treatment of patients with SARSCoV2 pneumonia [236].

\section{Anti-inflammatory agents}

\section{Tocilizumab}

Evidence has shown that patients with COVID-19 who were hospitalized in ICU had higher levels of plasma cytokines such as TNF- $\alpha$, granulocyte-colony stimulating factor (G-CSF), monocyte chemoattractant protein (MCP1), macrophage inflammatory protein $1 \alpha$ (MIP1 $\alpha$ ), interferon$\gamma$-inducible protein (IP10) and several interleukins including IL-6, IL-2, IL-7, and IL-10 [75, 217]. It is suggested that IL-6 plays an important role in the cytokine storm and drugs that target this cytokine are good treatment options for severe and critically ill COVID-19 patients. Tocilizumab, as a recombinant humanized anti-human IL-6 receptor monoclonal antibody, is mostly used in the treatment of rheumatoid arthritis. Via binding to sIL-6R (soluble form of IL-6 receptor) and mIL-6R (membrane-bound IL-6 receptor), it can inhibit the IL-6 signal transduction [237]. 
Tocilizumab is currently approved by the US FDA for the treatment of severe cytokine release syndrome (CRS) in addition to idiopathic arthritis, rheumatoid arthritis, and giant cell arteritis [238]. Several case report studies have been shown that tocilizumab improved some clinical symptoms in COVID-19 patients [239-244]. A pilot prospective open, single-arm multicenter study on off-label use of tocilizumab in 63 severe COVID-19 patients revealed improvement in respiratory and laboratory parameters [245].

The efficacy of tocilizumab for SARS-CoV2 was investigated in 20 severe or critically ill COVID-19 patients who received the drug in addition to conventional therapy. After a few days, the fever and other symptoms were shown to be improved significantly [246].

In one study, the impact of low-dose tocilizumab was assessed on mortality rate in 85 patients with COVID-19 related pneumonia and respiratory failure. Patients in the tocilizumab group revealed a significantly more survival rate than control patients. The important limitation of this study is the lack of a randomized double-blind approach [247]. Also, a study in a single academic medical center in the U.S. did not report significant clinical improvement in temperature or oxygen needs in most of the patients treated with tocilizumab. These data suggest that caution needs to be taken when using tocilizumab in severe COVID-19 patients [248].

Adverse effects of long-course treatment with tocilizumab consist of serious infections (such as pneumonia, urinary tract infection, cellulitis, etc.), gastrointestinal perforations, infusion reactions (such as hypertension, headache, and skin reactions), anaphylaxis, thrombocytopenia, elevated liver enzymes, and elevated lipid profile [238]. Adverse effects observed with tocilizumab use in patients with COVID-19 are also recorded $[249,250]$. The safety and efficacy of subcutaneous tocilizumab have been reported in a case series of patients with COVID-19 pneumonia. No significant adverse effects were seen except for a mild increase in the level of liver enzymes two days after administration with subsequent immediate normalization. Indeed, radiological findings and clinical symptoms were improved.[251].

Tocilizumab has been recommended for COVID-19 treatment in the Diagnosis and Treatment Protocol for Novel Coronavirus Pneumonia (Trial Version 7) promulgated by the Chinese government [252]. Also, thirty-two trials are currently evaluating the efficacy of tocilizumab for COVID19 (Table 2). Further controlled trial studies will be needed to clarify the true clinical effect of this IL-6-blocking therapy on COVID-19 infection and to determine optimal patient selection and timing for the use of tocilizumab during the disease process. Beyond proving its efficacy and above-mentioned side effects, it has several limitations such as being an expensive drug that can only be administered intravenously. So, more attention must be considered in future studies including the comparison of different dosages and modes of administration.

\section{Sarilumab}

Sarilumab (Kevzara $\left.{ }^{\circledR}\right)$ is an IL-6 receptor antagonist and has FDA approval in moderately to severely active rheumatoid arthritis adults who have inadequate response or intolerance to one or more disease-modifying anti-rheumatic drugs [253]. It has the potential to treat COVID-19 given the important role of IL-6 in the cytokine storm [254]. It binds to soluble and membrane-bound IL- 6 receptors. Because of the unavailability of IL-6 level measurements in most institutions, CRP levels may be useful for monitoring the response to therapy [143]. One ongoing randomized, double-blind, placebo-controlled study is comparing the effect of sarilumab higher dose (400 mg), sarilumab lower dose (200 mg), and placebo on 400 patients with COVID-19. Preliminary results from the phase II portion of an ongoing phase II/III sarilumab trial (NCT04315298) suggest that sarilumab can lower CRP in comparison to placebo. Also, sarilumab did not show a beneficial effect on the clinical outcomes of patients in "severe" disease [255]. Because of the lack of evidence for efficacy and safety of sarilumab in COVID-19 patients, this drug is not recommended currently. The clinical efficacy of sarilumab is being investigated in eight clinical trials which are illustrated in Table 2.

\section{Siltuximab}

Siltuximab (CNTO 328) is a monoclonal antibody-drug conjugate to interleukin-6 (IL-6), so it neutralizes IL-6 bioactivity. It also promotes tumor cell death and approved for the treatment of some viral diseases like HIV, human herpesvirus-8 (HHV-8), multicentric Castleman's disease (MCD), multiple myeloma (MM), myelodysplastic syndrome (MDS), prostate cancer, ovarian cancer, lung cancer and decrease anorexia and cachexia that related to cancer [256-258]. Siltuximab enhances cytochrome P450 activity and decreases the levels of TNF- $\alpha$, IL-1, CCL2, CXCL12, and VEGF [259, 260]. Currently, three studies (NCT04329650, NCT04322188, and NCT04330638) are investigating the effects of siltuximab on COVID-19 infections (Table 2).

\section{Eculizumab}

Eculizumab (Soliris ${ }^{\circledR}$ ) is a humanized monoclonal antibody that is commonly administered for the treatment of complement-mediated diseases [261,262]. The primary mechanism of eculizumab in these diseases is blocking the cleavage of complement protein C5 into C5a and C5b [262]. Both C5a and C5b have pro-inflammatory effects. Therefore, 
inflammation and cell damages would be decreased by suppressing the activity of this complement cascade [262]. Additionally, complement activity is associated with more progressing damage in patients with SARS-CoV-2 infection $[263,264]$. Based on the anti-complement mechanism of eculizumab, it is suggested to be an effective treatment for SARS-CoV2. However, despite its clinical usefulness, eculizumab increased the risk of some infections, especially meningococcal infection, even with prior vaccination [262, 265]. Therefore, a more conservative approach should be considered while we use this medication. In a case series study, four patients with severe pneumonia and ARDS were admitted and received eculizumab up to four doses along with standard treatment (lopinavir-ritonavir + hydroxychloroquine). All of the patients got better clinically and the treatment with eculizumab caused a reduction in inflammatory markers such as CRP [266]. The result of this study suggests the probable efficacy of eculizumab for the treatment of patients with pneumonia due to SARS-CoV2. To confirm the results of this study, some registered trials are ongoing. The selected doses of eculizumab in these studies are similar to other indications and are detailed in Table 2.

\section{Tetrandrine}

Tetrandrine (Tet) is a major alkaloid isolated from the roots of Stephania tetrandrae S. Moor of the Menispermaceae family. It has inhibitory effects on asthma, anti-allergic, anti-inflammatory, anti-oxidant, anti-fibrogenic activities, immunomodulation, and inhibition of platelet aggregation. Tetrandrine can improve the pulmonary function and structure by different mechanisms, including the modification of calcium metabolism in many cell types. Tetrandrine has potent inhibitory effects on IL-5 and IL-6 and prevents the production of IL-1. It has been reported that tetrandrine, as a nonspecific calcium channel blocker (CCB), has anti-inflammatory action through inhibition of 5-lipoxygenase [267, 268]. Tetrandrine with multiple mechanisms is also able to inhibit fibroblasts and pulmonary fibrosis. So, it may help improve lung function in COVID-19 patients. Currently, a clinical trial (NCT04308317) is in the process that investigates the effects of tetrandrine on pneumonia associated with COVID-19 (Table 2).

\section{NSAIDs}

Nonsteroidal anti-inflammatory drugs (NSAIDs) decrease lipid mediators, including leukotrienes, prostaglandins, and platelet-activating factors. Arachidonic acid is a precursor for the biosynthesis of leukotrienes and prostaglandins while lysophospholipid is the starting material for the synthesis of platelet-activating factor. Prostaglandins are products of cyclooxygenase-1 (COX-1) and COX-2; these enzymes are blocked by aspirin and ibuprofen. Because most proinflammatory prostaglandins are products of $\mathrm{COX}-2$, a selective COX-2 inhibitor such as celecoxib is the preferred anti-inflammatory agent. Although NSAIDs have some side effects, they are valuable drugs for treating life-threatening conditions, such as wet lungs [269].

Altogether, there are limited studies that investigate the effectiveness of NSAIDs on SARS-CoV2-associated pneumonia. Moreover, the role of NSAIDs during viral infections is still controversial. In vivo study showed that ibuprofen could induce overexpression of ACE2 [17]. This effect may theoretically increase susceptibility to SARS-CoV2 and also can worsen the clinical outcomes during COVID-19 [270]. Moreover, masking off the fever rises during SARS-CoV2, delaying in the laboratory diagnosis, and improper management of the infection may occur when using both NSAIDs and paracetamol (acetaminophen) [18]. Despite the effects of paracetamol as the first line of anti-pyretic drugs, some unverified reports exist on ibuprofen treatment in severe cases of SARS-CoV2 [9]. However, the administration of ibuprofen in children with poor fluid intake or suspected acute kidney injury is recommended to be avoided [235].

\section{Pulmonary vaso-effectors}

\section{Nitric oxide}

Nitric oxide (NO) is a selective pulmonary vasodilator gas used as rescue therapy in refractory hypoxemia due to ARDS [271]. Various ventilation methods, inhaled nitric oxide gas (iNO), surfactants, and extracorporeal life support cannot be used for every patient, because no evidence-based data exist [272]. In clinical settings, NO gas has been approved by the FDA for the treatment of pulmonary hypertension of the newborn in the presence of hypoxic respiratory failure. The NO gas has also been advocated as a rescue treatment in adults with hypoxic ARDS [273]. The reaction of NO with oxygen to form $\mathrm{NO}_{2}$ may cause inflammation in lung tissues. Moreover, oxidation of ferrous hemoglobin $(\mathrm{Hb})$ to methemoglobin (Met-Hb) by NO could reduce the oxygen transport and its release to tissues [274]. By carefully monitoring $\mathrm{NO} / \mathrm{NO}_{2}$ delivery and Met-Hb levels, the risk of iNO administration at $80 \mathrm{ppm}$ for $24 \mathrm{~h}$ can be minimal according to the FDA reports [275]. It has been shown in vitro and clinical studies that iNO has anti-viral activity against other strains of coronavirus [271]. The increased survival rate of infected eukaryotic cells by SARS-CoV1 using $S$-nitroso$\mathrm{N}$-acetylpenicillamine, as a NO donor, has shown the antiviral potential of NO [276]. Moreover, the use of iNO in patients with SARS-CoV1 who had severe symptoms could 
improve the severe hypoxemia and pulmonary hypertension, and shortened the time of ventilation support [276].

The very similar genomic identity of SARS-CoV1 to the SARS-CoV2 virus indicates the potential effectiveness of iNO therapy in patients with SARS-CoV2 [271].

Currently, some RCT studies are studying the effectiveness of iNO in mechanically ventilated patients with SARSCoV2 infection and some clinical improvements have also been seen in primary reports $[271,277]$.

\section{Sildenafil}

By inhibiting type 5 phosphodiesterase (PDE), sildenafil (Viagra ${ }^{\circledR}$ ) increases the level of cyclic guanosine monophosphate (cGMP) and induces vasodilatory effects in the pulmonary vasculature. Thus, it has FDA approval for the treatment of pulmonary arterial hypertension (PAH) in adults [278, 279].

According to the clinical trial study outlined in Table 2, a pilot study on the efficacy and safety of sildenafil citrate in ten patients with SARS-CoV2 infection is in progress [191]. No clear data are showing the effectiveness of sildenafil for COVID-19 until now.

\section{Aviptadil}

Aviptadil (VAP) is a synthetic vasoactive intestinal polypeptide (VIP), a synthetic form of the 28-amino-acid VIP, which increases the activity of adenosine cyclase. It causes cavernosal smooth muscle relaxation with a subsequent filling of cavernosal sinuses and erection [280].

Aviptadil reduces the right heart strain by acting as a weak pulmonary vasodilator. Moreover, it plays a role in the improvement of the oxygenation in patients with chronic lung disease and also can participate in a modest and shortlived pulmonary vasodilation induction without affecting systemic blood pressure [281]. Currently, two randomized clinical trials (NCT04311697 and NCT04360096) are in process that investigates the effects of aviptadil on acute lung injury, ARDS, and dyspnea associated with COVID-19 (Table 2). So, aviptadil might have an application in SARS$\mathrm{CoV} 2$ patients to improve lung function.

\section{Bevacizumab}

Bevacizumab (Avastin) is a humanized anti-VEGF (vascular endothelial growth factor) monoclonal antibody. Evidence has shown higher VEGF levels in patients with SARSCoV2. VEGF acts as an angiogenic inducer in vivo, so that bevacizumab by inhibiting VEGF can decrease the VEGF level caused by the up-regulation of the infected respiratory tract epithelium, severe inflammation, and hypoxia, all of which can decrease vascular permeability and pulmonary edema in patients with SARS-CoV2 [94, 282].

A pilot study of bevacizumab in the treatment of severe or critical Chinese patients with SARS-CoV2 pneumonia started in February 2020. Patients with SARS-CoV2 under ECG monitoring were given bevacizumab injection $500 \mathrm{mg}$ plus normal saline (NS) $100 \mathrm{ml}$, time is no less than $90 \mathrm{~min}$. $\mathrm{PaO} 2 / \mathrm{FiO} 2$ ratio at the times of $24 \mathrm{~h}, 72 \mathrm{~h}$, and 7 days is the main outcome of this pilot study [283]. In another multicenter triple-blind randomized placebo-controlled trial, the efficacy and safety of bevacizumab in 140 critical patients with SARS-CoV2 are evaluated in Qilu Hospital of Shandong University, China. Patients in the intervention group received bevacizumab $7.5 \mathrm{mg} / \mathrm{kg}$ body weight plus NS $100 \mathrm{ml}$. The time from randomization to an improvement of two points on a seven-category ordinal scale or live discharge from the hospital is considered as the main outcome [284].

\section{Losartan}

Losartan is an angiotensin II receptor antagonist that is used to treat hypertension and to slow long-term kidney damage in people with type 2 diabetes who also have high blood pressure. ACE2 has been identified as a host cell receptor that binds to viral surface proteins leading to infection [46]. Overactivation of ACE-AngII-AT1 caused by COVID-19 infection can lead to acute lung fibrosis. ACE2 has a determinant anti-fibrotic role in lung injury induced by sepsis, acid aspiration, SARS-CoV1, paraquat poisoning, and lethal avian influenza. The ACE-AngII-AT1 axis is also known as a molecular pathway for end-organ damage. Losartan may, thus, be suggested as a potential drug to protect lung fibrosis induced by coronavirus [285]. Currently, two clinical trials are in signs of progress that are investigating the beneficial effects of losartan in SARS-CoV2 infection compared to placebo (NCT04312009 and NCT04311177). In another case-control study, the effects of ACE-I and/or ARB drugs on the treatment of coronavirus disease are to be surveyed in 5000 patients (NCT04318418) (Table 2).

\section{Others}

\section{Carrimycin}

Carrimycin is an anti-mycobacterium drug used for treating tuberculosis especially in cases of drug resistance [286]. No clear data are showing the application of carrimycin for SARS-CoV2 infection until now. The efficacy and safety of carrimycin treatment in 520 patients with SARS-CoV2 are currently in progress as the multicenter, randomized, and open-controlled clinical trial study (NCT04286503, 
Table 2). The reversing of the fever to normal, resolving the pulmonary inflammation, and reduced SARS-CoV2 RNA levels at the end of treatment are the main primary outcomes.

\section{Mepolizumab}

Mepolizumab is a fully humanized IgG1 $\kappa$ monoclonal antibody that has affinity and selectivity to IL-5, thereby inhibiting ligand binding and decreasing peripheral blood eosinophils in humans. It is approved as an add-on therapy for patients with uncontrolled severe eosinophilic asthma [287]. Its potential for SARS-CoV2 is yet to be demonstrated.

\section{Conclusion}

The global pandemic of SARS-CoV2 in the face of a lack of effective prevention and therapeutic measures resulted in significant morbidity and mortality of the disease. Some clinical studies on conventional anti-viral therapies have been conducted around the world so far but the results remain to be controversial. In general, the selection of appropriate treatment for patients with COVID-19 depends on different factors, including the stage of the disease and its symptoms. Due to the differences in individual genetics in drug metabolism as well as the diversity of immune responses to the coronavirus, prescribing drugs among different patient populations may also be a challenge. Therefore, considering personalized medicine to select the best treatment along with an effective dose with minimal side effects would be a rational consideration in the future. Various studies are currently underway to evaluate vaccines against SARS-CoV2. However, due to genetic changes in the viral nucleic acid among different hosts, these specific vaccines may not have a definite preventive effect. Several therapies, which are ongoing by different clinical trial studies from January 2020 until now, can help to find more efficient treatment against SARSCoV2 with minimal side effects. Moreover, structural and molecular docking studies on virus proteins and different drug candidates may also play an important role in finding the specific target for therapeutic agents. Because of the contradictory benefit of anti-viral agents for COVID-19, the efficacy of drugs mostly was evaluated as the combination therapy. In the current condition, it seems that combination therapy of anti-viral drugs, including viral RNA polymerase inhibitors (such as remdesivir), viral protease inhibitors (such as atazanavir), blockers of virus entry to the host cell (such as arbidol), and immune system modulators (such as IVIg), as well as the use of supplement therapy (such as melatonin and vitamin D) can be recommended for effective control of COVID-19. Therefore, it is necessary to strive for developing specific drug/treatment and or vaccine, reduce the morbidity and mortality of SARS-CoV2 infection and establish scientific capabilities to rapidly diagnose and treat new viruses in the future.

Acknowledgements Authors have a special appreciation to BioRender team (BioRender.com) for creating the figure.

Funding Not applicable.

Data availability The datasets used and/or analyzed during the current study are available from the corresponding author on reasonable request.

\section{Compliance with ethical standards}

Conflict of in interests The authors have no conflict of interest financial, academic, commercial, political or personal.

Ethics approval and consent to participate Not applicable.

Consent for publication Not applicable.

\section{References}

1. Zhou P, et al. A pneumonia outbreak associated with a new coronavirus of probable bat origin. Nature. 2020;579:270-3.

2. De Salazar PM et al. Early Release-Identifying Locations with Possible Undetected Imported Severe Acute Respiratory Syndrome Coronavirus 2 Cases by Using Importation Predictions.

3. Organization WH. Naming the coronavirus disease (COVID19) and the virus that causes it. World Health Organization. 2020; https://wwwwhoint/emergencies/diseases/novel-coronavirus-2019/technical-guidance/naming-the-coronavirus-disease(covid-2019)-and-the-virus-that-causes-it.

4. Organization WH. Coronavirus disease 2019 (COVID-19)-Situation report-45; 2020

5. Gorbalenya AE, Baker SC, Baric R,Groot RJd, Drosten C, Gulyaeva AA, et al. Severe acute respiratory syndrome-related coronavirus: The species and its viruses-a statement of the Coronavirus Study Group. 2020. https://doi.org/10.1101/2020.02.07.937862.

6. Su S, et al. Epidemiology, genetic recombination, and pathogenesis of coronaviruses. Trends Microbiol. 2016;24(6):490-502.

7. Zumla A, et al. Coronaviruses - drug discovery and therapeutic options. Nat Rev Drug Discov. 2016;15(5):327.

8. Guo Y-R, et al. The origin, transmission and clinical therapies on coronavirus disease 2019 (COVID-19) outbreak-an update on the status. Military Med Res. 2020;7(1):1-10.

9. Cui J, Li F, Shi Z-L. Origin and evolution of pathogenic coronaviruses. Nat Rev Microbiol. 2019;17(3):181-92.

10. Li G, De Clercq E. Therapeutic options for the 2019 novel coronavirus (2019-nCoV). Berlin: Nature Publishing Group; 2020.

11. $\mathrm{Wu} \mathrm{C}$, et al. Analysis of therapeutic targets for SARS-CoV-2 and discovery of potential drugs by computational methods. Acta Pharmaceutica Sinica B. 2020;10:766-88.

12. Guan W.-J. et al. Clinical characteristics of coronavirus disease 2019 in China. New England J Med. 2020.

13. Dan H, Maureen G, Richard B. Quantitative mRNA expression proflling of ACE 2, a novel homologue of angiotensin converting enzyme. FEBS Lett. 2002;532(1-2):107. 
14. Letko M, Marzi A, Munster V. Functional assessment of cell entry and receptor usage for SARS-CoV-2 and other lineage B betacoronaviruses. Nat Microbiol. 2020;5:562-9.

15. Wang D, et al. Clinical characteristics of 138 hospitalized patients with 2019 novel coronavirus-infected pneumonia in Wuhan, China. JAMA. 2020;323:1061.

16. Lin L, et al. Hypothesis for potential pathogenesis of SARSCoV-2 infection- - a review of immune changes in patients with viral pneumonia. Emerg Microb Infect. 2020;9:727-32.

17. Wang L-S, et al. A review of the 2019 Novel Coronavirus (COVID-19) based on current evidence. Int J Antimicrob Agents. 2020;55:105948.

18. Favalli EG, et al. COVID-19 infection and rheumatoid arthritis: faraway, so close! Autoimmun Rev. 2020;19:102523.

19. Sahebnasagh, A. et al., Neutrophil Elastase Inhibitor (Sivelestat), may be a Promising Therapeutic Option for Management of Acute Lung Injury/Acute Respiratory Distress Syndrome or Disseminated Intravascular Coagulation in COVID-19. 2020; https://doi.org/10.22541/au.158981143.30930108.

20. National Health Commission of the People's Republic of China. Diagnosis and Treatment of Pneumonia Caused by 2019-nCoV (version 6). 2020.

21. Ge X-Y, et al. Isolation and characterization of a bat SARSlike coronavirus that uses the ACE2 receptor. Nature. 2013;503(7477):535-8.

22. Li W, et al. Angiotensin-converting enzyme 2 is a functional receptor for the SARS coronavirus. Nature. 2003;426(6965):450-4.

23. Omrani AS, et al. Ribavirin and interferon alfa-2a for severe Middle East respiratory syndrome coronavirus infection: a retrospective cohort study. Lancet Infect Dis. 2014;14(11):1090-5.

24. Zhang L, Liu Y. Potential interventions for novel coronavirus in China: a systematic review. J Med Virol. 2020;92(5):479-90.

25. Martinez MA. Compounds with therapeutic potential against novel respiratory 2019 coronavirus. Antimicrob Agents Chemother. 2020.

26. Zhang $\mathrm{Y}$ et al. Current targeted therapeutics against COVID-19: based on first-line experience in china. Pharmacol Res. 2020; p. 104854.

27. Khalili JS et al. Novel coronavirus treatment with ribavirin: groundwork for an evaluation concerning COVID-19. J Med Virol. 2020

28. Khan $\mathrm{S}$ et al. The emergence of a novel coronavirus (SARS$\mathrm{CoV}-2$ ), their biology and therapeutic options. J Clin Microbiol. 2020.

29. Khalid M, et al. Ribavirin and interferon- $\alpha 2 b$ as primary and preventive treatment for Middle East respiratory syndrome coronavirus: a preliminary report of two cases. Antivir Ther. 2015;20(1):87-91.

30. Kim UJ, et al. Combination therapy with lopinavir/ritonavir, ribavirin and interferon-alpha for Middle East respiratory syndrome. Antivir Ther. 2016;21(5):455-9.

31. Al-Tawfiq JA, et al. Ribavirin and interferon therapy in patients infected with the Middle East respiratory syndrome coronavirus: an observational study. Int J Infect Dis. 2014;20:42-6.

32. Arabi YM et al. Effect of Ribavirin and Interferon on the Outcome of Critically Ill patients with MERS. In C63. viral respiratory infections. American Thoracic Society. 2017; p. A6067-A6067.

33. Zhou C et al. COVID-19 with spontaneous pneumomediastinum. Lancet Infect Dis. 2020.

34. Alalwan AA, Taher A, Alaradi AH. A hemodialysis patient with severe COVID-19 pneumonia. Cureus. 2020;12(5):e7995.

35. Wang R et al. COVID-19 in hemodialysis patients: a report of 5 cases. Am J Kidney Dis. 2020.
36. Wu F, et al. Discontinuation of antiviral drugs may be the reason for recovered COVID-19 patients testing positive again. $\mathrm{Br}$ J Hosp Med. 2020;81(4):1-2.

37. Zhang P, et al. The novel coronavirus (COVID-19) pneumonia with negative detection of viral ribonucleic acid from nasopharyngeal swabs: a case report. BMC Infect Dis. 2020;20:1-7.

38. Hung IF-N, et al. Triple combination of interferon beta- $1 \mathrm{~b}$, lopinavir-ritonavir, and ribavirin in the treatment of patients admitted to hospital with COVID-19: an open-label, randomised, phase 2 trial. The Lancet. 2020.

39. Zeng Y-M, et al. Comparative effectiveness and safety of ribavirin plus interferon-alpha, lopinavir/ritonavir plus interferonalpha, and ribavirin plus lopinavir/ritonavir plus interferonalpha in patients with mild to moderate novel coronavirus disease 2019: study protocol. Chin Med J. 2020;133(9):1132-4.

40. Sarma P, et al. Therapeutic options for the treatment of 2019novel coronavirus: an evidence-based approach. Indian J Pharmacol. 2020;52(1):1.

41. Al-Tawfiq JA, Al-Homoud AH, Memish ZA. Remdesivir as a possible therapeutic option for the COVID-19. Travel Med Infect Dis.

42. Control CFD. Interim clinical guidance for management of patients with confirmed coronavirus disease (COVID-19). 2020.

43. Agostini ML, et al. Coronavirus susceptibility to the antiviral remdesivir (GS-5734) is mediated by the viral polymerase and the proofreading exoribonuclease. MBio. 2018;9(2):e00221-e318.

44. Sheahan TP, et al. Broad-spectrum antiviral GS-5734 inhibits both epidemic and zoonotic coronaviruses. Sci Trans Med. 2017;9(396):eaal3653.

45. Brown AJ, et al. Broad spectrum antiviral remdesivir inhibits human endemic and zoonotic deltacoronaviruses with a highly divergent RNA dependent RNA polymerase. Antiviral Res. 2019;169:104541.

46. Morse JS, Lalonde T, Xu S, Liu W. Learning from the past: possible urgent prevention and treatment options for severe acute respiratory infections caused by $2019-\mathrm{nCoV}$. Chembiochem. 2020;21(5):730-8. https://doi.org/10.1002/cbic.202000047.

47. Warren T, et al. Nucleotide prodrug GS-5734 is a broad-spectrum filovirus inhibitor that provides complete therapeutic protection against the development of Ebola virus disease (EVD) in infected non-human primates in Open Forum Infectious Diseases. Infect Dis Soc Am. 2015. https://doi.org/10.1093/ofid/ofv130.02.

48. Yethindra V. Role of GS-5734 (Remdesivir) in inhibiting SARSCoV and MERS-CoV: the expected role of GS-5734 (Remdesivir) in COVID-19 (2019-nCoV)-VYTR hypothesis. Int J Res Pharmaceutical Sci. 2020;11(SPL1):1-6.

49. Wang M, et al. Remdesivir and chloroquine effectively inhibit the recently emerged novel coronavirus $(2019-\mathrm{nCoV})$ in vitro. Cell Res. 2020;30(3):269-71.

50. Dong L, Hu S, Gao J. Discovering drugs to treat coronavirus disease 2019 (COVID-19). Drug Discov Therapeutics. 2020;14(1):58-60.

51. Al-Tawfiq JA, Al-Homoud AH, Memish ZA. Remdesivir as a possible therapeutic option for the COVID-19. Travel Med Infect Dis. 2020;34:101615.

52. Holshue ML, et al. First case of 2019 novel coronavirus in the United States. New Engl J Med. 2020.

53. Sheahan TP, et al. comparative therapeutic efficacy of remdesivir and combination lopinavir, ritonavir, and interferon beta against MERS-CoV. Nat Commun. 2020;11(1):1-14.

54. ClinicalTrials.gov identifier (NCT number): NCT04252664. 2020; Available From: https://clinicaltrials.gov/ct2/show/NCT04 252664 . 
55. ClinicalTrials.gov identifier (NCT number): NCT04257656. 2020; Available From: https://clinicaltrials.gov/ct2/show/NCT04 257656.

56. ClinicalTrials.gov identifier (NCT number): NCT04345276, Available From: https://clinicaltrials.gov/ct2/show/NCT0434527 $6 ?$ term $=$ NCT04345276\&cond $=$ COVID-19\&draw $=2 \&$ rank $=1$.

57. ClinicalTrials.gov identifier (NCT number): NCT04315948. 2020; Available From: https://clinicaltrials.gov/ct2/show/NCT04 315948.

58. ClinicalTrials.gov identifier (NCT number): NCT04292899. 2020; Available From: https://clinicaltrials.gov/ct2/show/NCT04 292899.

59. Goldman JD, et al. Remdesivir for 5 or 10 days in patients with severe Covid-19. New Engl J Med.

60. Shiraki K, Daikoku T. Favipiravir, an anti-influenza drug against life-threatening RNA virus infections. Pharmacol Therapeutics. 2020;209:107512.

61. Furuta Y, et al. T-705 (favipiravir) and related compounds: novel broad-spectrum inhibitors of RNA viral infections. Antiviral Res. 2009;82(3):95-102.

62. Guerrero NAS, et al. Favipiravir inhibits in vitro Usutu virus replication and delays disease progression in an infection model in mice. Antiviral Res. 2018;160:137-42.

63. Caroline AL, et al. Broad spectrum antiviral activity of favipiravir (T-705): protection from highly lethal inhalational Rift Valley Fever. PLoS Neglected Tropic Dis. 2014;8(4):e2790.

64. Hayden FG, Shindo N. Influenza virus polymerase inhibitors in clinical development. Curr Opin Infect Dis. 2019;32(2):176.

65. Rocha-Pereira J, et al. Favipiravir (T-705) inhibits in vitro norovirus replication. Biochem Biophys Res Commun. 2012;424(4):777-80.

66. Mentré F, et al. Dose regimen of favipiravir for Ebola virus disease. Lancet Infect Dis. 2015;15(2):150-1.

67. Rosenke $\mathrm{K}$, et al. Use of favipiravir to treat Lassa virus infection in macaques. Emerg Infect Dis. 2018;24(9):1696.

68. Safronetz D, et al. Antiviral efficacy of favipiravir against two prominent etiological agents of hantavirus pulmonary syndrome. Antimicrob Agents Chemother. 2013;57(10):4673-80.

69. Cai Q, et al. Experimental treatment with favipiravir for COVID19: an open-label control study. Engineering. 2020.

70. Chen C, et al. Favipiravir versus Arbidol for COVID-19: a randomized clinical trial. medRxiv, 2020.

71. Lu H. Drug treatment options for the 2019-new coronavirus (2019-nCoV). Biosci Trends. 2020;14(1):69-71.

72. Kamali A, Holodniy M. Influenza treatment and prophylaxis with neuraminidase inhibitors: a review. Infect Drug Resist. 2013;6:187

73. McCreary EK, Pogue JM. COVID-19 treatment: a review of early and emerging options. Open Forum Infect Dis. 2020. https://doi. org/10.1093/ofid/ofaa105.

74. Chen N, et al. Epidemiological and clinical characteristics of 99 cases of 2019 novel coronavirus pneumonia in Wuhan, China: a descriptive study. The Lancet. 2020;395(10223):507-13.

75. Xu X-W, et al. Clinical findings in a group of patients infected with the 2019 novel coronavirus (SARS-Cov-2) outside of Wuhan, China: retrospective case series. BMJ. 2020; 368.

76. Boffito $\mathrm{M}$, et al. Clinical use of lopinavir/ritonavir in a salvage therapy setting: pharmacokinetics and pharmacodynamics. Aids. 2002;16(15):2081-3.

77. Yao TT, et al. A systematic review of lopinavir therapy for SARS coronavirus and MERS coronavirus-a possible reference for coronavirus disease-19 treatment option. J Med Virol. 2020;92:556-63.

78. Yasri S, Wiwanitkit V. Dose prediction of lopinavir/ritonavir based on mathematic modeling for 2019-novel coronavirus
(2019-nCoV) infection. Asian Pacific J Trop Med. 2020;13(3):12. https://doi.org/10.4103/1995-7645.277815.

79. Que T, Wong V, Yuen K. Treatment of severe acute respiratory syndrome with lopinavir/ritonavir: a multicentre retrospective matched cohort study. Hong Kong Med J. 2003;9(6):399-406.

80. Chong YP, et al. Antiviral treatment guidelines for Middle East respiratory syndrome. Infect Chemother. 2015;47(3):212-22.

81. Mallat $\mathrm{J}$, et al. Hydroxychloroquine is associated with slower viral clearance in clinical COVID-19 patients with mild to moderate disease: a retrospective study. medRxiv. 2020.

82. Wiwanitkit S, Wiwanitkit V. Doubled dosage of sofosbuvir is expected for inhibiting Zika virus infection. Asian Pacific J Trop Med. 2017;10(6):612-3.

83. Corman VM, et al. Detection of 2019 novel coronavirus (2019$\mathrm{nCoV}$ ) by real-time RT-PCR. Eurosurveillance. 2020. https:// doi.org/10.2807/1560-7917.ES.2020.25.3.2000045.

84. Du Toit A. Outbreak of a novel coronavirus. Nat Rev Microbiol. 2020;18(3):123-123.

85. Wu P, et al. Real-time tentative assessment of the epidemiological characteristics of novel coronavirus infections in Wuhan, China, as at 22 January 2020. Eurosurveillance. 2020. https:// doi.org/10.2807/1560-7917.ES.2020.25.3.2000044.

86. Liu C, et al. Research and development on therapeutic agents and vaccines for COVID-19 and related human coronavirus diseases. Washington: ACS Publications; 2020.

87. Ortega JT, et al. Unrevealing sequence and structural features of novel coronavirus using in silico approaches: the main protease as molecular target. EXCLI J. 2020;19:400.

88. Wang Z, et al. Clinical characteristics and therapeutic procedure for four cases with 2019 novel coronavirus pneumonia receiving combined Chinese and Western medicine treatment. Biosci Trends. 2020;14:64-8.

89. Chu C, et al. Role of lopinavir/ritonavir in the treatment of SARS: initial virological and clinical findings. Thorax. 2004;59(3):252-6.

90. Pang J, Wang MX, Ang IYH, Tan SHX, Lewis RF, Chen JI-P, et al. Potential rapid diagnostics, vaccine and therapeutics for 2019 novel Coronavirus (2019-ncoV): a systematic review. J Clin Med. 2020;9(3):623. https://doi.org/10.3390/jcm9030623.

91. Lim J, et al. Case of the index patient who caused tertiary transmission of COVID-19 infection in Korea: the application of lopinavir/ritonavir for the treatment of COVID-19 infected pneumonia monitored by quantitative RT-PCR. J Korean Med Sci. 2020; 35(6).

92. Young BE, et al. Epidemiologic features and clinical course of patients infected with SARS-CoV-2 in Singapore. JAMA. 2020;323:1488.

93. Rosa SGV, Santos WC. Clinical trials on drug repositioning for COVID-19 treatment.

94. Cao B, et al. A trial of lopinavir-ritonavir in adults hospitalized with severe Covid-19. New Engl J Med. 2020.

95. Baden LR, Rubin EJ. Covid-19-the search for effective therapy. Mass Med Soc. 2020.

96. https://www.szdsyy.com/News/0a6c1e58-e3d0-4cd1. 22 Feb 2020.

97. Lopinavir and ritonavir. 6 Feb 2020.

98. Hurst M, Faulds D. Lopinavir. Drugs. 2000;60(6):1371-9.

99. Spanakis N, et al. Virological and serological analysis of a recent Middle East respiratory syndrome coronavirus infection case on a triple combination antiviral regimen. Int J Antimicrob Agents. 2014;44(6):528-32.

100. Kim UJ, et al. Case report Combination therapy with lopinavir/ ritonavir, ribavirin and interferon- $\alpha$ for Middle East respiratory syndrome. Antiviral Ther. 2016;21:455-9.

101. Arbidol and darunavir can effectively inhibit coronavirus. 21 Feb 2020. 
102. Mak E. Coronavirus outbreak pushes chinese biotech stocks higher despite market downturn. 7 Feb 2020.

103. Santos JR, et al. Simplification of antiretroviral treatment from darunavir/ritonavir monotherapy to darunavir/cobicistat monotherapy: effectiveness and safety in routine clinical practice. AIDS Res Hum Retroviruses. 2019;35(6):513-8.

104. Mathias A, et al. Pharmacokinetics and pharmacodynamics of GS-9350: a novel pharmacokinetic enhancer without anti-HIV activity. Clin Pharmacol Ther. 2010;87(3):322-9.

105. Hu F, Jiang J, Yin P. Prediction of potential commercially inhibitors against SARS-CoV-2 by multi-task deep model. arXiv preprint. 2020; arXiv:2003.00728.

106. Talluri S. Virtual High Throughput Screening Based Prediction of Potential Drugs for COVID-19. 2020.

107. Omotuyi OI, et al. Darunavir Disrupts Critical Nodes in Metastable 2019-nCoV-RBD/ACE-2 Complex. 2020.

108. Jiang Y, et al. Discovery of danoprevir (ITMN-191/R7227), a highly selective and potent inhibitor of hepatitis $\mathrm{C}$ virus (HCV) NS3/4A protease. J Med Chem. 2014;57(5):1753-69.

109. Wieslaw M. Kazmierski, Richard L. Jarvest, Jacob J. Plattner, Xianfeng Li. Linear and macrocyclic hepatitis $\mathrm{C}$ virus protease inhibitors. In: Levin JI editor. Macrocycles in drug discovery. vol 40. Royal Society of Chemistry; 2015. p. 247.

110. Zhavoronkov A, et al. Potential COVID-2019 3C-like protease inhibitors designed using generative deep learning approaches. Insilico Med Hong Kong Ltd A. 2020;307:E1.

111. Gorbalenya AE, et al. Cysteine proteases of positive strand RNA viruses and chymotrypsin-like serine protease. A distinct protein superfamily with a common structural fold. FEBS Lett. 1989;243(2):103-14.

112. Anand K, et al. Structure of coronavirus main proteinase reveals combination of a chymotrypsin fold with an extra alpha-helical domain. Embo J. 2002;21(13):3213-24.

113. Hosseini FS, Amanlou M. Simeprevir, potential candidate to repurpose for coronavirus infection: virtual screening and molecular docking study.

114. McCauley JA, Rudd MT. Hepatitis C virus NS3/4a protease inhibitors. Curr Opin Pharmacol. 2016;30:84-92.

115. Markham A, Keam SJ. Danoprevir: first global approval. Drugs. 2018;78(12):1271-6.

116. Gane EJ, et al. Efficacy and safety of danoprevir-ritonavir plus peginterferon Alfa-2a-ribavirin in hepatitis $\mathrm{C}$ virus genotype 1 prior null responders. Antimicrob Agents Chemother. 2014;58(2):1136-45.

117. Chen H., et al. First clinical study using HCV protease inhibitor danoprevir to treat naive and experienced COVID-19 Patients. MedRxiv. 2020.

118. Choi J, Horner KA, Carnevale K. Atazanavir, in StatPearls. StatPearls Publishing. 2019.

119. Anand K, et al. Structure of coronavirus main proteinase reveals combination of a chymotrypsin fold with an extra $\alpha$-helical domain. EMBO J. 2002;21(13):3213-24.

120. Borgio JF, et al. State-of-the-art tools unveil potent drug targets amongst clinically approved drugs to inhibit helicase in SARSCoV-2. Arch Med Sci. 2020;16(3):508.

121. Beck BR, et al. Predicting commercially available antiviral drugs that may act on the novel coronavirus (SARS-CoV-2) through a drug-target interaction deep learning model. Comput Struct Biotechnol J. 2020.

122. Atta MG, De Seigneux S, Lucas GM. Clinical pharmacology in HIV therapy. Clin J Am Soc Nephrol. 2019;14(3):435-44.

123. Contini A. Virtual screening of an FDA approved drugs database on two COVID-19 coronavirus proteins.

124. Shaghaghi,N. Molecular docking study of novel COVID-19 protease with low risk terpenoides compounds of plants. ChemRxiv. 2020 .
125. Bulduk I, Taktak F. Isolation and characterization of antitumor alkaloid from poppy capsules (papaver somniferum). J Chem. 2012; 2013.

126. Heydari M, Hashem Hashempur M, Zargaran A. Medicinal aspects of opium as described in Avicenna's Canon of Medicine. Acta medico-historica Adriatica. 2013;11(1):101-12.

127. Kumar D, et al. Understanding the binding affinity of noscapines with protease of SARS-CoV-2 for COVID-19 using MD simulations at different temperatures. J Biomol Struct Dyn. $2020 ; 1-14$.

128. Touret F, de Lamballerie X. Of chloroquine and COVID-19. Antiviral Res. 2020;177:104762.

129. Derwand R, Scholz M. Does zinc supplementation enhance the clinical efficacy of chloroquine/hydroxychloroquine to win todays battle against COVID-19? Med Hypotheses. 2020;142:109815.

130. Zhou D, Dai S-M, Tong Q. COVID-19: a recommendation to examine the effect of hydroxychloroquine in preventing infection and progression. J Antimicrob Chemother. 2020.

131. Yao X, et al. In vitro antiviral activity and projection of optimized dosing design of hydroxychloroquine for the treatment of severe acute respiratory syndrome coronavirus 2 (SARS-CoV-2). Clin Infect Dis. 2020

132. Patil VM, Singhal S, Masand N. A systematic review on use of aminoquinolines for the therapeutic management of COVID-19: Efficacy, safety and clinical trials. Life Sci. 2020;254:117775.

133. Gao J, Tian Z, Yang X. Breakthrough: Chloroquine phosphate has shown apparent efficacy in treatment of COVID-19 associated pneumonia in clinical studies. Biosci Trends. 2020.

134. [Expert consensus on chloroquine phosphate for the treatment of novel coronavirus pneumonia]. Zhonghua Jie $\mathrm{He} \mathrm{He} \mathrm{Hu} \mathrm{Xi} \mathrm{Za}$ Zhi. 2020; 43(3): 185-188.

135. Borba MGS, et al. Effect of high vs low doses of chloroquine diphosphate as adjunctive therapy for patients hospitalized with severe acute respiratory syndrome coronavirus 2 (SARSCoV-2) infection: a randomized clinical trial. JAMA Netw Open. 2020;3(4):e208857-e208857208857.

136. Chang R, Sun W-Z. Repositioning Chloroquine as Ideal Antiviral Prophylactic against COVID-19-Time is Now. 2020.

137. Shah S, et al. A systematic review of the prophylactic role of chloroquine and hydroxychloroquine in Coronavirus Disease-19 (COVID-19). Int J Rheumatic Dis. 2020.

138. de Novales FJM, et al. Early hydroxychloroquine is associated with an increase of survival in COVID-19 patients: an observational study. 2020.

139. Chen Z, et al. Efficacy of hydroxychloroquine in patients with COVID-19: results of a randomized clinical trial. MedRxiv 2020.

140. Mahevas M, et al. No evidence of clinical efficacy of hydroxychloroquine in patients hospitalized for COVID-19 infection with oxygen requirement: results of a study using routinely collected data to emulate a target trial. medrxiv, 2020.

141. Gautret $P$, et al. Hydroxychloroquine and azithromycin as a treatment of COVID-19: results of an open-label non-randomized clinical trial. Int J Antimicrob Agents. 2020; p. 105949.

142. Vijayvargiya $\mathrm{P}$, et al. Treatment considerations for COVID-19: a critical review of the evidence (or Lack Thereof) in Mayo clinic proceedings. Amsterdam: Elsevier; 2020.

143. Gautret $P$, et al. Clinical and microbiological effect of a combination of hydroxychloroquine and azithromycin in 80 COVID-19 patients with at least a six-day follow up: A pilot observational study. Travel Med Infect Dis. 2020; p. 101663.

144. Gevers S, et al. Safety considerations of chloroquine and hydroxychloroquine in treatment of COVID-19. Clin Microbiol Infecti. 2020.

145. Boriskin Y, et al. Arbidol: a broad-spectrum antiviral compound that blocks viral fusion. Curr Med Chem. 2008;15(10):997-1005. 
146. Blaising J, Polyak SJ, Pécheur E-I. Arbidol as a broad-spectrum antiviral: an update. Antiviral Res. 2014;107:84-94.

147. Haviernik J, et al. Arbidol (Umifenovir): a broad-spectrum antiviral drug that inhibits medically important arthropod-borne flaviviruses. Viruses. 2018;10(4):184.

148. Khamitov R, et al. Antiviral activity of arbidol and its derivatives against the pathogen of severe acute respiratory syndrome in the cell cultures. Vopr Virusol. 2008;53(4):9-13.

149. Notice on printing and distributing the diagnosis and treatment plan of COVID- 19 (trial version 6). National Health Commission of the People's Republic of China. 2020.

150. Deng L, et al. Arbidol combined with LPV/r versus LPV/r alone against Corona Virus Disease 2019: a retrospective cohort study. J Infect. 2020.

151. Genovese MC, et al. Baricitinib in patients with refractory rheumatoid arthritis. N Engl J Med. 2016;374:1243-52.

152. Choy EHS, et al. The effect of JAK1/JAK2 inhibition in rheumatoid arthritis: efficacy and safety of baricitinib. Clin Exp Rheumatol. 2019;37(4):694-70.

153. Richardson P, et al. Baricitinib as potential treatment for 2019$\mathrm{nCoV}$ acute respiratory disease. Lancet (London, England). 2020;395(10223):e30.

154. Favalli EG, et al. Baricitinib for COVID-19: a suitable treatment? Lancet Infect Dis 2020.

155. Cantini F, et al. Baricitinib therapy in COVID-19: A pilot study on safety and clinical impact. J Infect. 2020.

156. Mesa RA, Yasothan U, Kirkpatrick P. Ruxolitinib. Nature Publishing Group.

157. Albeituni S, et al. Mechanisms of action of ruxolitinib in murine models of hemophagocytic lymphohistiocytosis. Blood J Am Soc Hematol. 2019;134(2):147-59.

158. Mesa RA, et al. Development of a symptom assessment in patients with myelofibrosis: qualitative study findings. Health Quality Life Outcomes. 2019;17(1):61.

159. Cingam S, et al. Ruxolitinib in the treatment of polycythemia Vera: an update on health-related quality of life and patientreported outcomes. J Blood Med. 2019;10:381.

160. Stebbing J, et al. COVID-19: combining antiviral and antiinflammatory treatments. Lancet Infect Dis. 2020;20(4):400-2.

161. Zhang H, Baker A. Recombinant human ACE2: acing out angiotensin II in ARDS therapy. 2017.

162. Zhang H, et al. Angiotensin-converting enzyme 2 (ACE2) as a SARS-CoV-2 receptor: molecular mechanisms and potential therapeutic target. Intensive Care Med. 2020;46:586-90.

163. Scaglione F, Petrini O. Mucoactive agents in the therapy of upper respiratory airways infections: fair to describe them just as mucoactive? Clin Med Insights Ear Nose Throat. 2019; 12:1179550618821930.

164. Zanasi A, Mazzolini M, Kantar A. A reappraisal of the mucoactive activity and clinical efficacy of bromhexine. Multidiscip Respir Med. 2017;12:7.

165. Steinmetzer T, Hardes K. The antiviral potential of host protease inhibitors. In: Böttcher-Friebertshäuser E, Garten W, Klenk HD, editors. Activation of viruses by host proteases. Cham: Springer International Publishing; 2018. p. 279-325.

166. Li C-C, Wang X-J, Wang H-CR. Repurposing host-based therapeutics to control coronavirus and influenza virus. Drug Discovery Today. 2019;24(3):726-36.

167. Laporte M, Naesens L. Airway proteases: an emerging drug target for influenza and other respiratory virus infections. Curr Opin Virol. 2017;24:16-24.

168. Chen Y, et al. Structure analysis of the receptor binding of 2019nCoV. Biochem Biophys Res Commun. 2020.

169. Sun J, et al. COVID-19: epidemiology, evolution, and crossdisciplinary perspectives. Trends Mol Med. 2020.
170. Li C-C, Wang X-J, Wang H-CR. Repurposing host-based therapeutics to control coronavirus and influenza virus. Drug Discovery Today. 2019.

171. Pillaiyar T, Meenakshisundaram S, Manickam M. Recent discovery and development of inhibitors targeting coronaviruses. Drug Discovery Today. 2020.

172. Narayanan K, et al. Severe acute respiratory syndrome coronavirus nsp1 suppresses host gene expression, including that of type I interferon, in infected cells. J Virol. 2008;82(9):4471-9.

173. Kamitani W, et al. Severe acute respiratory syndrome coronavirus nsp1 protein suppresses host gene expression by promoting host mRNA degradation. Proc Natl Acad Sci. 2006;103(34):12885-90.

174. Loutfy MR, Blatt LM, Siminovitch KA, Ward S, Wolff B, Lho $\mathrm{H}$, et al. Interferon alfacon-1 plus corticosteroids in severe acute respiratory syndrome: a preliminary study. Jama. 2003;290(24):3222-8.

175. Lokugamage KG, et al. SARS-CoV-2 sensitive to type I interferon pretreatment. bioRxiv. 2020.

176. Guidelines for the Prevention, Diagnosis, and Treatment of Novel Coronavirus-induced Pneumonia, The 6th edn. 23 Feb 2020.

177. $\mathrm{Xu} \mathrm{Z}$, et al. Pathological findings of COVID-19 associated with acute respiratory distress syndrome. Lancet Respir Med. 2020.

178. Han W, et al. The course of clinical diagnosis and treatment of a case infected with coronavirus disease 2019. J Med Virol. 2020

179. Song Y, et al. SARS-CoV-2 induced diarrhoea as onset symptom in patient with COVID-19. Gut. 2020.

180. Shetty R, et al. Therapeutic opportunities to manage COVID-19/ SARS-CoV-2 infection: present and future. Indian J Ophthalmol. 2020;68(5):693.

181. Nile SH, et al. COVID-19: Pathogenesis, cytokine storm and therapeutic potential of interferons. Cytokine Growth Factor Rev. 2020.

182. Estebanez M, et al. Clinical evaluation of IFN beta1b in COVID19 pneumonia: a retrospective study. medRxiv. 2020.

183. Yuen K-S, et al. SARS-CoV-2 and COVID-19: The most important research questions. Cell Biosci. 2020;10(1):1-5.

184. Morgenstern B, et al. Ribavirin and interferon- $\beta$ synergistically inhibit SARS-associated coronavirus replication in animal and human cell lines. Biochem Biophys Res Commun. 2005;326(4):905-8.

185. Dattola V, et al. Relationship between interferon beta-1A administration and intracranial vascular tone regulation in patients with relapsing-remitting multiple sclerosis: a pilot study. BioMed Res Int. 2017; 2017.

186. Callahan MK, Wolchok JD. At the Bedside: CTLA-4- and PD1-blocking antibodies in cancer immunotherapy. J Leukocyte Biol. 2013;94(1):41-53.

187. Liu B, Song Y, Liu D. Recent development in clinical applications of PD-1 and PD-L1 antibodies for cancer immunotherapy. J Hematol Oncol. 2017;10(1):1-8.

188. Brahmamdam $\mathrm{P}$, et al. Delayed administration of anti-PD-1 antibody reverses immune dysfunction and improves survival during sepsis. J Leukoc Biol. 2010;88(2):233-40.

189. Chiappelli F, Khakshooy A, Greenberg G. CoViD-19 immunopathology and immunotherapy. Bioinformation. 2020;16(3):219-22.

190. Weber J, et al. Safety profile of nivolumab monotherapy: a pooled analysis of patients with advanced melanoma. J Clin Oncol. 2017.

191. Renoux GR. The general immunopharmacology of levamisole. Drugs. 1980;20(2):89-99.

192. Joffe M, Sukha N, Rabson A. Lymphocyte subsets in measles. Depressed helper/inducer subpopulation reversed by in vitro treatment with levamisole and ascorbic acid. J Clin Investig. 1983;72(3):971-80. 
193. Rosenthal M, Trabert U, Müller W. The effect of Levamisole on peripheral blood lymphocyte subpopulations in patients with rheumatoid arthritis and ankylosing spondylitis. Clin Exp Immunol. 1976;25(3):493.

194. Iranian Clinical Trial Register. Identifier: IRCT20200516047468N1, Available From: https://www.irct.ir/ trial/46561

195. Iranian Clinical Trial Register. Identifier: IRCT20190917044805N2, Available From: https://www.irct.ir/ trial/46561

196. Li T, Lu H, Zhang W. Clinical observation and management of COVID-19 patients. Emerg Microb Infect. 2020;9(1):687-90.

197. Belouzard S, et al. Mechanisms of coronavirus cell entry mediated by the viral spike protein. Viruses. 2012;4(6):1011-33.

198. Stockman LJ, Bellamy R, Garner P. SARS: systematic review of treatment effects. PLoS Med. 2006;3(9):e343.

199. Wu W, et al. Clinical features of 96 patients with severe acute respiratory syndrome from a hospital outbreak. Zhonghua nei ke za zhi. 2003;42(7):453-7.

200. Li Z, et al. Clinical analysis of pediatric SARS cases in Beijing. Zhonghua er ke za zhi= Chin J Pediatrics. 2003;41(8):574-7.

201. Xu Y, et al. Characteristics of pediatric SARS-CoV-2 infection and potential evidence for persistent fecal viral shedding. Nat Med. 2020;26(4):502-5.

202. Duan K, et al. Effectiveness of convalescent plasma therapy in severe COVID-19 patients. Proc Natl Acad Sci. 2020;117(17):9490-6.

203. Driggin E, et al. Cardiovascular considerations for patients, health care workers, and health systems during the COVID-19 pandemic. J Am College Cardiol. 2020;75(18):2352-71.

204. Portaccio E. Evidence-based assessment of potential use of fingolimod in treatment of relapsing multiple sclerosis. Core Evidence. 2011;6:13.

205. Sato K, et al. Disease exacerbation after the cessation of fingolimod treatment in Japanese patients with multiple sclerosis. Intern Med. 2018; p. 0793-18.

206. ClinicalTrials.gov identifier (NCT number): NCT04268537, Available From: https://clinicaltrials.gov/ct2/show/NCT0426853 7 ?term $=$ NCT0 $4268537 \&$ cond $=$ COVID $-19 \&$ draw $=2 \&$ rank $=1$

207. $\mathrm{Xu} \mathrm{H}$, et al. Systemic injection of thalidomide prevent and attenuate neuropathic pain and alleviate neuroinflammatory response in the spinal dorsal horn. J Pain Res. 2019;12:3221.

208. Hada M. Chemotherapeutic Strategy with Synbiotics, Thalidomide and Celecoxib for severe COVID-19 Pneumonia. Association between microbiota, chronic inflammation and pneumonia. 2020.

209. Chen C, et al. Thalidomide combined with low-dose glucocorticoid in the treatment of COVID-19 Pneumonia. 2020.

210. Thompson BT. Glucocorticoids and acute lung injury. Crit Care Med. 2003;31(4):S253-S257257.

211. Cain DW, Cidlowski JA. Immune regulation by glucocorticoids. Nat Rev Immunol. 2017;17(4):233.

212. Rhen T, Cidlowski JA. Antiinflammatory action of glucocorticoids-new mechanisms for old drugs. N Engl J Med. 2005;353(16):1711-23.

213. Wang Y, et al. Early, low-dose and short-term application of corticosteroid treatment in patients with severe COVID-19 pneumonia: single-center experience from Wuhan, China. medRxiv. 2020.

214. Lee N, et al. Effects of early corticosteroid treatment on plasma SARS-associated Coronavirus RNA concentrations in adult patients. J Clin Virol. 2004;31(4):304-9.

215. Arabi YM, et al. Corticosteroid therapy for critically ill patients with Middle East respiratory syndrome. Am J Respir Crit Care Med. 2018;197(6):757-67.
216. Huang C, et al. Clinical features of patients infected with 2019 novel coronavirus in Wuhan, China. The Lancet. 2020;395(10223):497-506.

217. Alfaraj SH, et al. Clinical predictors of mortality of Middle East Respiratory Syndrome Coronavirus (MERS-CoV) infection: A cohort study. Travel Med Infect Dis. 2019;29:48-50.

218. Booth CM, et al. Clinical features and short-term outcomes of 144 patients with SARS in the greater Toronto area. JAMA. 2003;289(21):2801-9.

219. Yam LY-C, et al. Corticosteroid treatment of severe acute respiratory syndrome in Hong Kong. J Infect. 2007;54(1):28-39.

220. Azimi S, et al. Corticosteroids administration following COVID19-induced acute respiratory distress syndrome. Is it harmful or life-saving? Adv J Emerg Med. 2020;4(2s):e43.

221. Lewis SR, et al. Pharmacological agents for adults with acute respiratory distress syndrome. Cochrane Database Syst Rev. 2019; (7).

222. Columbus C, Brust KB, Arroliga AC. 2019 novel coronavirus: an emerging global threat. In: Baylor University Medical Center Proceedings. Milton Park: Taylor \& Francis; 2020.

223. Russell CD, Millar JE, Baillie JK. Clinical evidence does not support corticosteroid treatment for 2019-nCoV lung injury. The Lancet. 2020;395(10223):473-5.

224. Shang L, et al. On the use of corticosteroids for 2019-nCoV pneumonia. The Lancet. 2020;395(10225):683-4.

225. WHO. Clinical management of severe acute respiratory infection when novel coronavirus (nCoV) infection is suspected. 2020.

226. Khot WY, Nadkar MY. The 2019 Novel Coronavirus outbreak-a global threat. J Assoc Physicians India. 2020;68(3):67.

227. Zhou Z-G, et al. Short-term moderate-dose corticosteroid plus immunoglobulin effectively reverses COVID-19 patients who have failed low-dose therapy. 2020.

228. Wu C, et al. Risk factors associated with acute respiratory distress syndrome and death in patients with coronavirus disease 2019 pneumonia in Wuhan, China. JAMA Int Med 2020

229. Horby PLW Emberson J, Mafham M, Bell J, Linsell L, Staplin N, Brightling C, Ustianowski A, Elmahi E, Prudon B. Effect of dexamethasone in hospitalized patients with COVID-19: preliminary report. 2020.

230. Yuan $\mathrm{H}$, et al. A current emerging respiratory infection: epidemiological and clinical characteristics, diagnosis and treatments of COVID-19. Diagnosis and treatments of COVID-19 (3/6/2020). 2020.

231. Munck A, Guyre PM. Glucocorticoid physiology, pharmacology and stress. Steroid hormone resistance. Berlin: Springer; 1986. p. 81-96.

232. Zhou Y-H, et al. Effectiveness of glucocorticoid therapy in patients with severe novel coronavirus pneumonia: protocol of a randomized controlled trial. Chin Med J. 2020

233. Zhou W, et al. Potential benefits of precise corticosteroids therapy for severe 2019-nCoV pneumonia. Signal Trans Targeted Therapy. 2020;5(1):1-3.

234. Sinha I. Guidance for the clinical management of children admitted to hospital with suspected COVID-19. British Paediatric Respiratory Society. 2020.

235. Matsuyama S, et al. The inhaled corticosteroid ciclesonide blocks coronavirus RNA replication by targeting viral NSP15. bioRxiv. 2020.

236. Kaly L, Rosner I. Tocilizumab-A novel therapy for non-organspecific autoimmune diseases. Best Pract Res Clin Rheumatol. 2012;26(1):157-65.

237. Zhang S, et al. Rational use of Tocilizumab in the treatment of novel coronavirus pneumonia. Clin Drug Invest. 2020; p. 1-8.

238. Zhang X, et al. First case of COVID-19 in a patient with multiple myeloma successfully treated with tocilizumab. Blood Adv. 2020;4(7):1307. 
239. De Luna G, et al. Rapid and severe Covid-19 pneumonia with severe acute chest syndrome in a sickle cell patient successfully treated with tocilizumab. Am J Hematol. 2020.

240. Michot J-M, et al. Tocilizumab, an anti-IL6 receptor antibody, to treat Covid-19-related respiratory failure: a case report. Ann Oncol. 2020.

241. Coyle J, et al. A recovered case of COVID-19 myocarditis and ARDS treated with corticosteroids, tocilizumab, and experimental AT-001. JACC. 2020.

242. Cellina M, et al. Favorable changes of CT findings in a patient with COVID-19 pneumonia after treatment with tocilizumab. Diagn Interv Imaging. 2020;101(5):323-4.

243. Radbel J, Narayanan N, Bhatt PJ. Use of tocilizumab for COVID-19 infection-induced cytokine release syndrome: a cautionary case report. Chest. 2020;158(1):e15-9.

244. Sciascia $S$, et al. Pilot prospective open, single-arm multicentre study on off-label use of tocilizumab in severe patients with COVID-19. Clin Exp Rheumatol. 2020; 8.

245. Xu X, et al. Effective treatment of severe COVID-19 patients with Tocilizumab. ChinaXiV. 2020;202003:v1.

246. Capra R, et al. Impact of low dose tocilizumab on mortality rate in patients with COVID-19 related pneumonia. Eur $\mathrm{J}$ Intern Med. 2020.

247. Rimland CA, et al. Clinical characteristics and early outcomes in patients with COVID-19 treated with tocilizumab at a United States academic center. medRxiv. 2020.

248. Toniati $\mathrm{P}$, et al. Tocilizumab for the treatment of severe COVID-19 pneumonia with hyperinflammatory syndrome and acute respiratory failure: a single center study of 100 patients in Brescia, Italy. Autoimmun Rev. 2020; p. 102568.

249. Vikse J, Henry BM. Tocilizumab in COVID-19: beware of risk of intestinal perforation. Int J Antimicrob Agents. 2020.

250. Mazzitelli $\mathrm{M}$, et al. Use of subcutaneous tocilizumab in patients with COVID-19 pneumonia. J Med Virol. 2020.

251. https://www.nhc.gov.cn/yzygj/s7653p/202003/46c9294a7d fe4cef80dc7f5912eb1989/files/ce3e6945832a438eaae415350 a8ce964.pdf. 2020.

252. Arnaldez FI, O’Day SJ, Drake CG, Fox BA, Fu B, Urba WJ, et al. The Society for Immunotherapy of Cancer perspective on regulation of interleukin-6 signaling in COVID-19-related systemic inflammatory response. J immunother cancer. 2020;8(1):e000930.

253. Clerkin KJ, et al. Coronavirus disease 2019 (COVID-19) and cardiovascular disease. Circulation. 2020.

254. Guida JP. Chloroquine, Hydroxychloroquine and Covid19: a systematic review of literature. Int Am J Med Health. 2020;3:1-10.

255. Chen R, Chen B. Siltuximab (CNTO 328): a promising option for human malignancies. Drug Design Dev Ther. 2015;9:3455.

256. Markham A, Patel T. Siltuximab: first global approval. Drugs. 2014;74(10):1147-52.

257. Van Rhee F, et al. Siltuximab for multicentric Castleman's disease: a randomised, double-blind, placebo-controlled trial. Lancet Oncol. 2014;15(9):966-74.

258. Yao $\mathrm{X}$, et al. Targeting interleukin-6 in inflammatory autoimmune diseases and cancers. Pharmacol Ther. 2014;141(2):125-39.

259. Coward J, et al. Interleukin-6 as a therapeutic target in human ovarian cancer. Clin Cancer Res. 2011;17(18):6083-96.

260. Martí-Carvajal AJ, et al. Eculizumab for treating patients with paroxysmal nocturnal hemoglobinuria. Cochrane Database Syst Rev (10).

261. Dubois EA, Cohen AF. Eculizumab. Br J Clin Pharmacol. 2009;68(3):318.

262. Song YG, Shin H-S. COVID-19, A clinical syndrome manifesting as hypersensitivity pneumonitis. Infect Chemother. 2020;52:110.
263. Guo R-F, Ward PA. Role of C5a in inflammatory responses. Annu Rev Immunol. 2005;23:821-52.

264. Struijk G, et al. Meningococcal sepsis complicating eculizumab treatment despite prior vaccination. Am J Transplant. 2013;13(3):819-20.

265. Diurno F, et al. Eculizumab treatment in patients with COVID19: preliminary results from real life ASL Napoli 2 Nord experience. Eur Rev Med Pharmacol Sci. 2020;24(7):4040-7.

266. Choi H-S, et al. Anti-inflammatory effects of fangchinoline and tetrandrine. J Ethnopharmacol. 2000;69(2):173-9.

267. Xie Q-M, et al. Pharmacological actions of tetrandrine in inflammatory pulmonary diseases. Acta Pharmacol Sin. 2002;23(12):1107-13.

268. Chen S. Pathophysiology-based mechanism and management strategies for deadly leaking lungs caused by 2019 novel coronavirus.

269. Fang L, Karakiulakis G, Roth M. Are patients with hypertension and diabetes mellitus at increased risk for COVID-19 infection? Lancet Respir Med. 2020.

270. Lei C, et al. Protocol of a randomized controlled trial testing inhaled Nitric Oxide in mechanically ventilated patients with severe acute respiratory syndrome in COVID-19 (SARS-CoV-2). medRxiv. 2020.

271. De Luca D. Managing neonates with respiratory failure due to SARS-CoV-2. Lancet Child Adolescent Health. 2020.

272. Gebistorf F, et al. Inhaled nitric oxide for acute respiratory distress syndrome (ARDS) in children and adults. Cochrane Database Syst Rev. 2016; (6).

273. Wright RO, Lewander WJ, Woolf AD. Methemoglobinemia: etiology, pharmacology, and clinical management. Ann Emerg Med. 1999;34(5):646-56.

274. Feiner JR, Bickler PE. Improved accuracy of methemoglobin detection by pulse CO-oximetry during hypoxia. Anesth Analg. 2010;111(5):1160-7.

275. Chen L, et al. Inhalation of nitric oxide in the treatment of severe acute respiratory syndrome: a rescue trial in Beijing. Clin Infect Dis. 2004;39(10):1531-5.

276. Berra L, et al. Protocol for a randomized controlled trial testing inhaled nitric oxide therapy in spontaneously breathing patients with COVID-19. medRxiv. 2020.

277. Barst RJ, et al. Pulmonary arterial hypertension: a comparison between children and adults. Eur Respir J. 2011;37(3):665-77.

278. Cohen AH, et al. Inhibition of cyclic 3'-5'-guanosine monophosphate-specific phosphodiesterase selectively vasodilates the pulmonary circulation in chronically hypoxic rats. J Clin Investig. 1996;97(1):172-9.

279. Duncan C, et al. Erectile dysfunction: a global review of intracavernosal injectables. World J Urol. 2019;37(6):1007-144.

280. Leuchte $\mathrm{HH}$, et al. Inhalation of vasoactive intestinal peptide in pulmonary hypertension. Eur Respir J. 2008;32(5):1289-94.

281. Ferrara N, Hillan KJ, Novotny W. Bevacizumab (Avastin), a humanized anti-VEGF monoclonal antibody for cancer therapy. Biochem Biophys Res Commun. 2005;333(2):328-35.

282. ClinicalTrials.gov identifier (NCT number): NCT04355494, Available From: https://clinicaltrials.gov/ct2/show/NCT0435549 4? term $=$ NCT04355494\& cond $=$ COVID $-19 \&$ draw $=2 \&$ rank $=1$

283. ClinicalTrials.gov identifier (NCT number): NCT04346797, Available From: https://clinicaltrials.gov/ct2/show/NCT0434679 7? term $=$ NCT04346797\& cond $=$ COVID-19\&draw $=2 \&$ rank $=1$

284. Zeinalian $\mathrm{M}$, et al. A potential protective role of losartan against coronavirus-induced lung damage. Infect Control Hosp Epidemiol. 2020;41(6):752-3.

285. Wang Y, et al. Use of carrimycin in mycobacterium tuberculosis infection resistance. Google Patents.

286. Hillas G, Fouka E, Papaioannou AI. Antibodies targeting the interleukin-5 signaling pathway used as add-on therapy for 
patients with severe eosinophilic asthma: a review of the mechanism of action, efficacy, and safety of the subcutaneously administered agents, mepolizumab and benralizumab. Expert Rev Respir Med. 2020;14(4):353-65.

287. Markham A, Patel T. Siltuximab: first global approval. Drugs. 2014;74(10):1147-52.

288. Yao X, et al. Targeting interleukin-6 in inflammatory autoimmune diseases and cancers. Pharmacol Therapeutics. 2014;141(2):125-39.

289. ClinicalTrials.gov identifier (NCT number): NCT04280588, Available From: https://clinicaltrials.gov/ct2/show/NCT0428058 8 ?term $=$ NCT04280588 \& cond $=$ COVID $-19 \&$ draw $=2 \&$ rank $=1$. 2020.

290. ClinicalTrials.gov identifier (NCT number): NCT04305106, Available From: https://clinicaltrials.gov/ct2/show/ NCT04305106?term $=$ NCT04305106\& cond $=$ COVID $-19 \&$ draw $=2 \&$ rank $=1.2020$.

291. ClinicalTrials.gov identifier (NCT number): NCT04275414, Available From: https://clinicaltrials.gov/ct2/show/ NCT04 275414?term=NCT04275414\& cond=COVID $-19 \&$ draw $=2 \&$ rank $=1.2020$

292. ClinicalTrials.gov identifier (NCT number): NCT04329650, Available From: https://clinicaltrials.gov/ct2/show/NCT04 329650. 2020.

293. ClinicalTrials.gov identifier (NCT number): NCT04377750, Available From: https://clinicaltrials.gov/ct2/show/NCT04 377750. 2020.

294. ClinicalTrials.gov identifier (NCT number): NCT04377503, Available From: https://clinicaltrials.gov/ct2/show/NCT04 377503. 2020.

295. ClinicalTrials.gov identifier (NCT number): NCT04321993, Available From: https://clinicaltrials.gov/ct2/show/ NCT04321993?term=NCT04321993\& cond=COVID $-19 \&$ draw $=2 \&$ rank $=1$

296. ClinicalTrials.gov identifier (NCT number): NCT04320277, Available From: https://clinicaltrials.gov/ct2/show/ NCT0432027 7?term $=$ NCT0432027 $\&$ cond $=$ COVID $-19 \&$ draw $=2 \&$ rank $=1$

297. ClinicalTrials.gov identifier (NCT number): NCT04345289, Available From: https://clinicaltrials.gov/ct2/show/NCT0434528 9 ?term $=$ NCT04345289\&cond $=$ COVID- $19 \&$ draw $=2 \&$ rank $=1$

298. ClinicalTrials.gov identifier (NCT number): NCT04268537, Available From: https://clinicaltrials.gov/ct2/show/NCT0426853 $7 ?$ term $=$ NCT04268537 \& cond $=$ COVID-19\&draw $=2 \&$ rank $=1$. 2020.

299. ClinicalTrials.gov identifier (NCT number): NCT04366206, Available From: https://clinicaltrials.gov/ct2/show/NCT0436620 $6 ?$ term $=$ NCT04366206 \& cond $=$ COVID- $19 \&$ draw $=2 \&$ rank $=1$

300. ClinicalTrials.gov identifier (NCT number): NCT04287686, Available From: https://clinicaltrials.gov/ct2/show/ NCT04 287686 ? term $=$ NCT04 $287686 \&$ cond $=$ COVID $-19 \&$ draw $=2 \&$ rank $=1.2020$.

301. ClinicalTrials.gov identifier (NCT number): NCT04264533, Available From: https://clinicaltrials.gov/ct2/show/NCT0426453 3 ?term $=$ NCT04264533\& cond $=$ COVID-19\&draw $=2 \&$ rank $=1$. 2020.

302. ClinicalTrials.gov identifier (NCT number): NCT04323514, Available From: https://clinicaltrials.gov/ct2/show/NCT0432351 4 ? term $=$ NCT04323514\&cond $=$ COVID $-19 \&$ draw $=2 \&$ rank $=1$. 2020.

303. ClinicalTrials.gov identifier (NCT number): NCT04310228, Available From: https://clinicaltrials.gov/ct2/show/NCT0431022 8 ?term $=$ NCT04310228 \& cond $=$ COVID-19\&draw $=2 \&$ rank $=1$. 2020.

304. ClinicalTrials.gov identifier (NCT number): NCT04319900, Available From: https://clinicaltrials.gov/ct2/show/NCT0431990
$0 ?$ term $=$ NCT04319900 $\&$ cond $=$ COVID-19\&draw $=2 \&$ rank $=1$. 2020.

305. Chinese Clinical Trial Register, Identifier: ChiCTR2000029600, Available From: https://www.chictr.org.cn/showprojen .aspx? proj=49042. 2020.

306. Chen C, et al. Favipiravir versus arbidol for COVID-19: a randomized clinical trial. MedRxiv.

307. Chinese Clinical Trial Register, Identifier: ChiCTR2000029544, Available From: https://www.chictr.org.cn/showprojen .aspx? proj=49013. 2020.

308. Chinese Clinical Trial Register, Identifier: ChiCTR2000030113, Available From: www.chictr.org.cn/hvshowproject. aspx?id=22695. 2020.

309. ClinicalTrials.gov identifier (NCT number): NCT04273529, Available From: https://clinicaltrials.gov/ct2/show/NCT0427352 9? term $=$ NCT04273529\&draw $=2 \&$ rank $=1.2020$.

310. ClinicalTrials.gov identifier (NCT number): NCT04312009, Available From: https://clinicaltrials.gov/ct2/show/NCT0431200 9? term $=$ NCT04312009\&draw $=2 \&$ rank $=1.2020$.

311. ClinicalTrials.gov identifier (NCT number): NCT04311177, Available From: https://clinicaltrials.gov/ct2/show/NCT0431117 7. 2020.

312. ClinicalTrials.gov identifier (NCT number): NCT04318418, Available From: https://clinicaltrials.gov/ct2/show/NCT0431841 8. 2020.

313. ClinicalTrials.gov identifier (NCT number): NCT04261270, Available From: https://clinicaltrials.gov/ct2/show/NCT0426127 0?term $=$ NCT04261270\&draw $=2 \&$ rank $=1.2020$.

314. Chinese Clinical Trial Register. Identifier: ChiCTR2000029387, Available From: https://www.chictr.org.cn/showprojen .aspx? ?proj=48782. 2020.

315. Chinese Clinical Trial Register. Identifier: ChiCTR2000029803, Available From: https://www.chictr.org.cn/showprojen .aspx?proj=49428. 2020 .

316. Chinese Clinical Trial Register. Identifier: ChiCTR2000029740, Available From: https://www.chictr.org.cn/showprojen .aspx?proj=49317. 2020.

317. Chinese Clinical Trial Register. Identifier: ChiCTR2000029898, Available From: https://www.chictr.org.cn/showprojen .aspx?proj=49482. 2020.

318. Chinese Clinical Trial Register. Identifier: ChiCTR2000029899, Available From: https://www.chictr.org.cn/showprojen .aspx?proj=49536. 2020.

319. Chinese Clinical Trial Register. Identifier: ChiCTR2000030054, Available From: https://www.chictr.org.cn/showprojen .aspx?proj=49869. 2020.

320. Chinese Clinical Trial Register. Identifier: ChiCTR2000029765, Available From: https://www.chictr.org.cn/showprojen aspx?proj=49409. 2020

321. Chinese Clinical Trial Register. Identifier: ChiCTR2000030894, Available From: https://www.chictr.org.cn/showprojen .aspx?proj=51126. 2020.

322. Chinese Clinical Trial Register. Identifier: ChiCTR2000029542, Available From: https://www.chictr.org.cn/showprojen .aspx?proj=48968. 2020.

323. Chinese Clinical Trial Register. Identifier: ChiCTR2000030031, Available From: https://www.chictr.org.cn/showprojen .aspx? proj=49806. 2020.

324. Chinese Clinical Trial Register. Identifier: ChiCTR2000031204, Available From: www.chictr.org.cn/hvshowproject. aspx $? \mathrm{id}=24756.2020$.

325. Chinese Clinical Trial Register. Identifier: ChiCTR2000030718, Available From: https://www.chictr.org.cn/showprojen .aspx?proj=50843. 2020. 
326. Chinese Clinical Trial Register. Identifier: ChiCTR2000030417, Available From: https://www.chictr.org.cn/showprojen .aspx?proj=50279. 2020.

327. Chinese Clinical Trial Register. Identifier: ChiCTR2000030987, Available From: https://www.chictr.org.cn/showprojen .aspx?proj=48919. 2020.

328. Chinese Clinical Trial Register. Identifier: ChiCTR2000029559, Available From: https://www.chictr.org.cn/showprojen .aspx? ?proj=48880. 2020.

329. Chinese Clinical Trial Register. Identifier: ChiCTR2000029609, Available From: https://www.chictr.org.cn/showprojen .aspx?proj=49145. 2020.

330. Chinese Clinical Trial Register. Identifier: ChiCTR2000029741, Available From: https://www.chictr.org.cn/showprojen .aspx?proj=49263. 2020.

331. Chinese Clinical Trial Register. Identifier: ChiCTR2000029760, Available From: www.chictr.org.cn/hvshowproject. aspx $? i d=22820.2020$.

332. Chinese Clinical Trial Register. Identifier: ChiCTR2000029761, Available From: https://www.chictr.org.cn/showprojen .aspx?proj=49400. 2020.

333. Chinese Clinical Trial Register. Identifier: ChiCTR2000029762, Available From: www.chictr.org.cn/hvshowproject. aspx?id=22816. 2020.

334. Chinese Clinical Trial Register. Identifier: ChiCTR2000029826, Available From: www.chictr.org.cn/hvshowproject. aspx?id=23666. 2020.

335. Chinese Clinical Trial Register. Identifier: ChiCTR2000029837, Available From: https://www.chictr.org.cn/showprojen .aspx? proj=49495. 2020.

336. Chinese Clinical Trial Register. Identifier: ChiCTR2000029868, Available From: https://www.chictr.org.cn/showprojen aspx?proj=49524. 2020.

337. Chinese Clinical Trial Register. Identifier: ChiCTR2000029935, Available From: https://apps.who.int/trialsearch/Trial 2.aspx?TrialID $=$ ChiCTR2000029935. 2020.

338. Chinese Clinical Trial Register. Identifier: ChiCTR2000029939, Available From: https://www.chictr.org.cn/showprojen .aspx?proj=49612. 2020.

339. Chinese Clinical Trial Register. Identifier: ChiCTR2000029975, Available From: www.chictr.org.cn/hvshowproject. aspx id $=22801.2020$.

340. Chinese Clinical Trial Register. Identifier: ChiCTR2000029988, Available From: https://www.chictr.org.cn/showprojen .aspx?proj=49218. 2020.

341. Chinese Clinical Trial Register. Identifier: ChiCTR2000029992, Available From: https://www.chictr.org.cn/showprojen .aspx?proj=49574. 2020.

342. ClinicalTrials.gov identifier (NCT number): NCT04343768, Available From: https://clinicaltrials.gov/ct2/show/NCT0434376 8. 2020.

343. ClinicalTrials.gov identifier (NCT number): NCT04350671, Available From: https://clinicaltrials.gov/ct2/show/NCT0435067 1. 2020.

344. ClinicalTrials.gov identifier (NCT number): NCT04350281, Available From: https://clinicaltrials.gov/ct2/show/NCT0435028 1. 2020.

345. ClinicalTrials.gov identifier (NCT number): NCT04356677, Available From: https://clinicaltrials.gov/ct2/show/NCT0435667 7. 2020.

346. ClinicalTrials.gov identifier (NCT number): NCT04392427, Available From: https://clinicaltrials.gov/ct2/show/NCT0439242 7. 2020 .

347. ClinicalTrials.gov identifier (NCT number): NCT04276688, Available From: https://clinicaltrials.gov/ct2/show/NCT0427668 8. 2020.
348. ClinicalTrials.gov identifier (NCT number): NCT04323527, Available From: https://clinicaltrials.gov/ct2/show/NCT0432352 7. 2020.

349. ClinicalTrials.gov identifier (NCT number): NCT04261517, Available From: https://clinicaltrials.gov/ct2/show/NCT0426151 7. 2020.

350. ClinicalTrials.gov identifier (NCT number): NCT04316377, Available From: https://clinicaltrials.gov/ct2/show/NCT0431637 7. 2020.

351. ClinicalTrials.gov identifier (NCT number): NCT04308668, Available From: https://clinicaltrials.gov/ct2/show/NCT0430866 8. 2020.

352. ClinicalTrials.gov identifier (NCT number): NCT04323631, Available From: https://clinicaltrials.gov/ct2/show/NCT0432363 1. 2020.

353. ClinicalTrials.gov identifier (NCT number): NCT04315896, Available From: https://clinicaltrials.gov/ct2/show/NCT04 315896. 2020.

354. ClinicalTrials.gov identifier (NCT number): NCT04318444, Available From: https://clinicaltrials.gov/ct2/show/NCT04 318444. 2020.

355. ClinicalTrials.gov identifier (NCT number): NCT04318015, Available From: https://clinicaltrials.gov/ct2/show/NCT04 318015. 2020.

356. ClinicalTrials.gov identifier (NCT number): NCT04303299, Available From: https://clinicaltrials.gov/ct2/show/ NCT04303299?term=NCT04303299\&cond=COVID $-19 \&$ draw $=2 \&$ rank $=1.2020$.

357. ClinicalTrials.gov identifier (NCT number): NCT04303507, Available From: https://clinicaltrials.gov/ct2/show/NCT04 303507. 2020.

358. ClinicalTrials.gov identifier (NCT number): NCT04322396, Available From: https://clinicaltrials.gov/ct2/show/NCT04 322396. 2020.

359. ClinicalTrials.gov identifier (NCT number): NCT04322123, Available From: https://clinicaltrials.gov/ct2/show/NCT04 322123. 2020.

360. ClinicalTrials.gov identifier (NCT number): NCT04321278, Available From: https://clinicaltrials.gov/ct2/show/NCT04 321278. 2020.

361. ClinicalTrials.gov identifier (NCT number): NCT04315298, Available From: https://clinicaltrials.gov/ct2/show/NCT04 315298. 2020.

362. ClinicalTrials.gov identifier (NCT number): NCT04322773, Available From: https://clinicaltrials.gov/ct2/show/NCT04 322773. 2020.

363. ClinicalTrials.gov identifier (NCT number): NCT04317092, Available From: https://clinicaltrials.gov/ct2/show/NCT04 317092. 2020.

364. ClinicalTrials.gov identifier (NCT number): NCT04315480, Available From: https://clinicaltrials.gov/ct2/show/NCT04 315480. 2020

365. ClinicalTrials.gov identifier (NCT number): NCT04320615, Available From: https://clinicaltrials.gov/ct2/show/NCT04 320615. 2020.

366. ClinicalTrials.gov identifier (NCT number): NCT04306705, Available From: https://clinicaltrials.gov/ct2/show/NCT04 306705. 2020.

367. ClinicalTrials.gov identifier (NCT number): NCT04313023, Available From: https://clinicaltrials.gov/ct2/show/NCT04 313023. 2020.

368. ClinicalTrials.gov identifier (NCT number): NCT04312997, Available From: https://clinicaltrials.gov/ct2/show/NCT04 312997. 2020 
369. Iranian Clinical Trial Register. Identifier: IRCT20100228003449N29, Available From: https://www.irct. $\mathrm{ir} / \mathrm{trial} / 46567.2020$.

370. Iranian Clinical Trial Register. Identifier: IRCT20160625028622N1, Available From: https://www.irct. $\mathrm{ir} / \mathrm{trial} / 46576.2020$.

371. Iranian Clinical Trial Register. Identifier: IRCT20200128046294N2, Available From: https://www.irct. $\mathrm{ir} / \mathrm{trial} / 46463.2020$.

372. Iranian Clinical Trial Register. Identifier: IRCT20100228003449N30, Available From: https://www.irct. ir/trial/46536. 2020.

373. ClinicalTrials.gov identifier (NCT number): NCT04311697, Available From: https://clinicaltrials.gov/ct2/show/NCT04 311697. 2020

374. ClinicalTrials.gov identifier (NCT number): NCT04360096, Available From: https://clinicaltrials.gov/ct2/show/NCT0436009 6. 2020.

375. ClinicalTrials.gov identifier (NCT number): NCT04275245, Available From: https://clinicaltrials.gov/ct2/show/NCT0427524 5. 2020.

376. ClinicalTrials.gov identifier (NCT number): NCT04322682, Available From: https://clinicaltrials.gov/ct2/show/NCT0432268 2. 2020.

377. ClinicalTrials.gov identifier (NCT number): NCT04322565, Available From: https://clinicaltrials.gov/ct2/show/NCT0432256 5. 2020.

378. ClinicalTrials.gov identifier (NCT number): NCT04308317, Available From: https://clinicaltrials.gov/ct2/show/NCT0430831 7. 2020.

379. ClinicalTrials.gov identifier (NCT number): NCT04330638, Available From: https://clinicaltrials.gov/ct2/show/NCT0433063 8. 2020.

380. ClinicalTrials.gov identifier (NCT number): NCT04322188, Available From: https://clinicaltrials.gov/ct2/show/NCT0432218 8. 2020.

381. ClinicalTrials.gov identifier (NCT number): NCT04321096, Available From: https://clinicaltrials.gov/ct2/show/NCT0432109 6. 2020.

382. Chinese Clinical Trial Register. Identifier: ChiCTR2000029308, Available From: https://www.chictr.org.cn/showprojen .aspx?proj=48684. 2020.

383. Chinese Clinical Trial Register. Identifier: ChiCTR2000029468, Available From: https://www.chictr.org.cn/showprojen .aspx?proj=48919. 2020.

384. Chinese Clinical Trial Register. Identifier: ChiCTR2000029539, Available From: https://www.chictr.org.cn/showprojen .aspx?proj=48991. 2020.

385. Chinese Clinical Trial Register. Identifier: ChiCTR2000029541, Available From: https://www.chictr.org.cn/showprojen .aspx?proj=48992. 2020.

386. Chinese Clinical Trial Register. Identifier: ChiCTR2000029548, Available From: https://www.chictr.org.cn/showprojen .aspx?proj=49015. 2020.

387. Chinese Clinical Trial Register. Identifier: ChiCTR2000029603, Available From: https://www.chictr.org.cn/showprojen .aspx?proj=49075. 2020.

388. Chinese Clinical Trial Register. Identifier: ChiCTR2000029621, Available From: https://www.chictr.org.cn/showprojen .aspx ?proj=49165. 2020.

389. Chinese Clinical Trial Register. Identifier: ChiCTR2000029638, Available From: www.chictr.org.cn/hvshowproject. aspx?id=33516. 2020.

390. Chinese Clinical Trial Register. Identifier: ChiCTR2000029656, Available From: https://www.chictr.org.cn/showprojen .aspx?proj=49086. 2020 .
391. Chinese Clinical Trial Register. Identifier: ChiCTR2000029759, Available From: https://apps.who.int/trialsearch/Trial 2.aspx?TrialID=ChiCTR2000029759. 2020.

392. Chinese Clinical Trial Register. Identifier: ChiCTR2000029989, Available From: https://www.chictr.org.cn/showprojen .aspx?proj=49720. 2020.

393. Chinese Clinical Trial Register. Identifier: ChiCTR2000030013, Available From: https://apps.who.int/trialsearch/Trial 2.aspx?TrialID=ChiCTR2000030013. 2020.

394. Chinese Clinical Trial Register. Identifier: ChiCTR2000030187, Available From: https://www.chictr.org.cn/showprojen .aspx?proj=50057. 2020.

395. Chinese Clinical Trial Register. Identifier: ChiCTR2000030262 , Available From: https://www.chictr.org.cn/showprojen aspx?proj=50136

396. Chinese Clinical Trial Register. Identifier: ChiCTR2000030442, Available From: https://apps.who.int/trialsearch/Trial 2.aspx?TrialID=ChiCTR2000030442. 2020.

397. Chinese Clinical Trial Register. Identifier: ChiCTR2000030480 , Available From: https://www.chictr.org.cn/showprojen .aspx?proj=50470.

398. Chinese Clinical Trial Register. Identifier: ChiCTR2000031782 , Available From: https://www.chictr.org.cn/showprojen .aspx?proj=51934.

399. Chinese Clinical Trial Register. Identifier: ChiCTR2000030481 , Available From: https://www.chictr.org.cn/showprojen aspx?proj=50453.

400. Chinese Clinical Trial Register. Identifier: ChiCTR2000030922, Available From: www.chictr.org.cn/hvshowproject. aspx?id=24034. 2020.

401. Chinese Clinical Trial Register. Identifier: ChiCTR2000031196 , Available From: https://www.chictr.org.cn/showprojen .aspx?proj=51112.

402. Iranian Clinical Trial Register. Identifier: IRCT20151227025726N12, Available From: https://www.irct. $\mathrm{ir} /$ trial/46561. 2020.

403. ClinicalTrials.gov identifier (NCT number): NCT04244591, Available From: https://clinicaltrials.gov/ct2/show/NCT0424459 1. 2020 .

404. ClinicalTrials.gov identifier (NCT number): NCT04251871, Available From: https://clinicaltrials.gov/ct2/show/NCT0425187 1. 2020.

405. ClinicalTrials.gov identifier (NCT number): NCT04252274, Available From: https://clinicaltrials.gov/ct2/show/NCT0425227 4 ?term $=$ NCT04252274\&draw $=2 \&$ rank $=1.2020$.

406. ClinicalTrials.gov identifier (NCT number): NCT04254874, Available From: https://clinicaltrials.gov/ct2/show/NCT0425487 4. 2020.

407. ClinicalTrials.gov identifier (NCT number): NCT04255017, Available From: https://clinicaltrials.gov/ct2/show/NCT0425501 7?term $=$ NCT04255017\&draw $=2 \&$ rank $=1.2020$.

408. ClinicalTrials.gov identifier (NCT number): NCT04260594, Available From: https://clinicaltrials.gov/ct2/show/NCT0426059 4. 2020.

409. ClinicalTrials.gov identifier (NCT number): NCT04261426, Available From: https://clinicaltrials.gov/ct2/show/NCT0426142 6. 2020.

410. ClinicalTrials.gov identifier (NCT number): NCT04261907, Available From: https://clinicaltrials.gov/ct2/show/NCT0426190 7. 2020.

411. ClinicalTrials.gov identifier (NCT number): NCT04264858, Available From: https://clinicaltrials.gov/ct2/show/NCT0426485 8. 2020.

412. ClinicalTrials.gov identifier (NCT number): NCT04273581, Available From: https://clinicaltrials.gov/ct2/show/NCT0427358 1 ?term $=$ NCT04273581\&draw $=2 \&$ rank $=1.2020$. 
413. ClinicalTrials.gov identifier (NCT number): NCT04275388, Available From: https://clinicaltrials.gov/ct2/show/NCT0427538 8. 2020.

414. ClinicalTrials.gov identifier (NCT number): NCT04280705, Available From: https://clinicaltrials.gov/ct2/show/NCT0428070 5. 2020.

415. ClinicalTrials.gov identifier (NCT number): NCT04286503, Available From: https://clinicaltrials.gov/ct2/show/NCT0428650 3. 2020.

416. ClinicalTrials.gov identifier (NCT number): NCT04292730, Available From: https://clinicaltrials.gov/ct2/show/NCT0429273 0. 2020.

417. ClinicalTrials.gov identifier (NCT number): NCT04293887, Available From: https://clinicaltrials.gov/ct2/show/NCT0429388 7. 2020.

418. ClinicalTrials.gov identifier (NCT number): NCT04295551, Available From: https://clinicaltrials.gov/ct2/show/NCT0429555 1. 2020.

419. ClinicalTrials.gov identifier (NCT number): NCT04302766, Available From: https://clinicaltrials.gov/ct2/show/NCT0430276 6. 2020.

420. ClinicalTrials.gov identifier (NCT number): NCT04304053, Available From: https://clinicaltrials.gov/ct2/show/NCT0430405 3? term $=$ NCT04304053\&draw $=2 \&$ rank $=1.2020$.

421. ClinicalTrials.gov identifier (NCT number): NCT04304313, Available From: https://clinicaltrials.gov/ct2/show/NCT0430431 3. 2020.

422. ClinicalTrials.gov identifier (NCT number): NCT04305457, Available From: https://clinicaltrials.gov/ct2/show/NCT0430545 7. 2020.

423. ClinicalTrials.gov identifier (NCT number): NCT04306393, Available From: https://clinicaltrials.gov/ct2/show/NCT0430639 3. 2020.

424. ClinicalTrials.gov identifier (NCT number): NCT04307693, Available From: https://clinicaltrials.gov/ct2/show/NCT0430769 3. 2020.

425. ClinicalTrials.gov identifier (NCT number): NCT04312243, Available From: https://clinicaltrials.gov/ct2/show/NCT0431224 3. 2020.

426. ClinicalTrials.gov identifier (NCT number): NCT04320238, Available From: https://clinicaltrials.gov/ct2/show/NCT0432023 8. 2020.

427. ClinicalTrials.gov identifier (NCT number): NCT04321174, Available From: https://clinicaltrials.gov/ct2/show/NCT0432117 4. 2020

428. ClinicalTrials.gov identifier (NCT number): NCT04321616, Available From: https://clinicaltrials.gov/ct2/show/NCT0432161 6. 2020.

429. ClinicalTrials.gov identifier (NCT number): NCT04323592, Available From: https://clinicaltrials.gov/ct2/show/NCT0432359 2. 2020.

430. ClinicalTrials.gov identifier (NCT number): NCT04323761, Available From: https://clinicaltrials.gov/ct2/show/NCT0432376 1. 2020.

431. ClinicalTrials.gov identifier (NCT number): NCT04325633, Available From: https://clinicaltrials.gov/ct2/show/NCT0432563 3. 2020.

432. ClinicalTrials.gov identifier (NCT number): NCT04340050, Available From: https://clinicaltrials.gov/ct2/show/NCT0434005 0. 2020.

433. ClinicalTrials.gov identifier (NCT number): NCT04358614, Available From: https://clinicaltrials.gov/ct2/show/NCT0435861 4. 2020.

434. ClinicalTrials.gov identifier (NCT number): NCT04365764, Available From: https://clinicaltrials.gov/ct2/show/NCT0436576 4 ?term $=$ NCT04365764\& cond $=$ COVID-19\&draw $=2 \&$ rank $=1$
435. ClinicalTrials.gov identifier (NCT number): NCT04273763, Available From: https://clinicaltrials.gov/ct2/show/NCT0427376 3 ?term $=$ NCT04273763\&cond $=$ COVID-19\&draw $=2 \&$ rank $=1$

436. ClinicalTrials.gov identifier (NCT number): NCT04355026, Available From: https://clinicaltrials.gov/ct2/show/NCT0435502 6 term $=$ NCT0 $3555026 \&$ cond $=$ COVID $-19 \&$ draw $=2 \&$ rank $=1$

437. ClinicalTrials.gov identifier (NCT number): NCT04340349, Available From: https://clinicaltrials.gov/ct2/show/NCT0434034 9? term $=$ NCT04340349\& cond $=$ COVID-19\&draw $=2 \&$ rank $=1$

438. ClinicalTrials.gov identifier (NCT number): NCT04345276, Available From: https://clinicaltrials.gov/ct2/show/NCT0434527 6. 2020.

439. ClinicalTrials.gov identifier (NCT number): NCT04346797, Available From: https://clinicaltrials.gov/ct2/show/NCT0434679 7. 2020.

440. ClinicalTrials.gov identifier (NCT number): NCT04355494, Available From: https://clinicaltrials.gov/ct2/show/NCT0435549 4. 2020.

441. ClinicalTrials.gov identifier (NCT number): NCT04382950, Available From: https://clinicaltrials.gov/ct2/show/NCT0438295 0 ?term $=$ NCT0 $4382950 \&$ cond $=$ COVID $-19 \&$ draw $=2 \&$ rank $=1$

442. ClinicalTrials.gov identifier (NCT number): NCT04363216, Available From: https://clinicaltrials.gov/ct2/show/NCT0436321 $6 ?$ term $=$ NCT04363216\& cond $=$ COVID-19\&draw $=2 \&$ rank $=1$

443. ClinicalTrials.gov identifier (NCT number): NCT04395768, Available From: https://clinicaltrials.gov/ct2/show/NCT0439576 8 ?term $=$ NCT04395768 \& cond $=$ COVID $-19 \&$ draw $=2 \&$ rank $=1$

444. ClinicalTrials.gov identifier (NCT number): NCT04342728, Available From: https://clinicaltrials.gov/ct2/show/NCT0434272 8 ?term $=$ NCT0 $342728 \&$ cond $=$ COVID $-19 \&$ draw $=2 \&$ rank $=1$

445. ClinicalTrials.gov identifier (NCT number): NCT04357782, Available From: https://clinicaltrials.gov/ct2/show/NCT0435778 2 ?term $=$ NCT04357782 \& cond $=$ COVID $-19 \&$ draw $=2 \&$ rank $=1$

446. ClinicalTrials.gov identifier (NCT number): NCT04344184, Available From: https://clinicaltrials.gov/ct2/show/NCT0434418 4 ?term $=$ NCT0 $344184 \&$ cond $=$ COVID $-19 \&$ draw $=2 \&$ rank $=1$

447. ClinicalTrials.gov identifier (NCT number): NCT03680274, Available From: https://clinicaltrials.gov/ct2/show/NCT0368027 4? term $=$ NCT03680274\& cond $=$ COVID $-19 \&$ draw $=2 \&$ rank $=1$

448. Iranian Clinical Trial Register. Identifier: IRCT20200324046850N5, Available From: https://www.irct.ir/ trial/46561.

449. Iranian Clinical Trial Register. Identifier: IRCT20200411047025N1, Available From: https://www.irct.ir/ trial/47078. 2020.

450. ClinicalTrials.gov identifier (NCT number): NCT04373044, Available From: https://clinicaltrials.gov/ct2/show/NCT0437304 4 ? term $=$ NCT04373044\& cond $=$ COVID-19\&draw $=2 \&$ rank $=1$

451. ClinicalTrials.gov identifier (NCT number): NCT04340232, Available From: https://clinicaltrials.gov/ct2/show/NCT0434023 2 ?term $=$ NCT04340232\& cond $=$ COVID-19\&draw $=2 \&$ rank $=1$.

452. ClinicalTrials.gov identifier (NCT number): NCT04393051, Available From: https://clinicaltrials.gov/ct2/show/NCT0439305 1 ?term $=$ NCT04393051\&cond $=$ COVID-19\&draw $=2 \&$ rank $=1$.

453. ClinicalTrials.gov identifier (NCT number): NCT04362943, Available From: https://clinicaltrials.gov/ct2/show/NCT0436294 3 ?term $=$ NCT0 $362943 \&$ cond $=$ COVID $-19 \&$ draw $=2 \&$ rank $=1$

454. ClinicalTrials.gov identifier (NCT number): NCT04390464, Available From: https://clinicaltrials.gov/ct2/show/NCT0439046 4? term $=$ NCT0 $4390464 \&$ cond $=$ COVID $-19 \&$ draw $=2 \&$ rank $=1$

455. ClinicalTrials.gov identifier (NCT number): NCT04346147, Available From: https://clinicaltrials.gov/ct2/show/NCT0434614 7 ?term $=$ NCT04346147\& cond $=$ COVID-19\&draw $=2 \&$ rank $=1$

456. ClinicalTrials.gov identifier (NCT number): NCT04331470, Available From: https://clinicaltrials.gov/ct2/show/NCT0433147 0. 2020. 
457. ClinicalTrials.gov identifier (NCT number): NCT04360122, Available From: https://clinicaltrials.gov/ct2/show/NCT0436012 2. 2020.

458. ClinicalTrials.gov identifier (NCT number): NCT04383717, Available From: https://clinicaltrials.gov/ct2/show/NCT0438371 7. 2020.

459. ClinicalTrials.gov identifier (NCT number): NCT04362332, Available From: https://clinicaltrials.gov/ct2/show/NCT0436233 2. 2020.

460. ClinicalTrials.gov identifier (NCT number): NCT04328493, Available From: https://clinicaltrials.gov/ct2/show/NCT0432849 3. 2020.

461. ClinicalTrials.gov identifier (NCT number): NCT04333628, Available From: https://clinicaltrials.gov/ct2/show/NCT0433362 8. 2020.

462. ClinicalTrials.gov identifier (NCT number): NCT04344951, Available From: https://clinicaltrials.gov/ct2/show/NCT0434495 1. 2020.

463. ClinicalTrials.gov identifier (NCT number): NCT04331600, Available From: https://clinicaltrials.gov/ct2/show/NCT0433160 0. 2020.

464. ClinicalTrials.gov identifier (NCT number): NCT04353336, Available From: https://clinicaltrials.gov/ct2/show/NCT0435333 6. 2020.

465. ClinicalTrials.gov identifier (NCT number): NCT04333732, Available From: https://clinicaltrials.gov/ct2/show/NCT0433373 2. 2020.

466. ClinicalTrials.gov identifier (NCT number): NCT04360759, Available From: https://clinicaltrials.gov/ct2/show/NCT0436075 9. 2020.

467. ClinicalTrials.gov identifier (NCT number): NCT04342650, Available From: https://clinicaltrials.gov/ct2/show/NCT0434265 0. 2020.

468. ClinicalTrials.gov identifier (NCT number): NCT04333914, Available From: https://clinicaltrials.gov/ct2/show/NCT0433391 4. 2020.

469. ClinicalTrials.gov identifier (NCT number): NCT04351191, Available From: https://clinicaltrials.gov/ct2/show/NCT0435119 1. 2020.

470. ClinicalTrials.gov identifier (NCT number): NCT04346667, Available From: https://clinicaltrials.gov/ct2/show/NCT0434666 7. 2020.

471. ClinicalTrials.gov identifier (NCT number): NCT04349371, Available From: https://clinicaltrials.gov/ct2/show/NCT0434937 1. 2020.

472. ClinicalTrials.gov identifier (NCT number): NCT04341727, Available From: https://clinicaltrials.gov/ct2/show/NCT0434172 7. 2020.

473. ClinicalTrials.gov identifier (NCT number): NCT04359316, Available From: https://clinicaltrials.gov/ct2/show/NCT0435931 6. 2020.

474. ClinicalTrials.gov identifier (NCT number): NCT04334148, Available From: https://clinicaltrials.gov/ct2/show/NCT0433414 8. 2020.

475. ClinicalTrials.gov identifier (NCT number): NCT04324463, Available From: https://clinicaltrials.gov/ct2/show/NCT0432446 3. 2020.

476. ClinicalTrials.gov identifier (NCT number): NCT04342221, Available From: https://clinicaltrials.gov/ct2/show/NCT0434222 1. 2020.

477. ClinicalTrials.gov identifier (NCT number): NCT04351620, Available From: https://clinicaltrials.gov/ct2/show/NCT0435162 0. 2020.

478. ClinicalTrials.gov identifier (NCT number): NCT04385264, Available From: https://clinicaltrials.gov/ct2/show/NCT0438526 4. 2020.
479. ClinicalTrials.gov identifier (NCT number): NCT04329832, Available From: https://clinicaltrials.gov/ct2/show/NCT0432983 2. 2020 .

480. ClinicalTrials.gov identifier (NCT number): NCT04334382, Available From: https://clinicaltrials.gov/ct2/show/NCT0433438 2. 2020.

481. ClinicalTrials.gov identifier (NCT number): NCT04361318, Available From: https://clinicaltrials.gov/ct2/show/NCT0436131 8. 2020.

482. ClinicalTrials.gov identifier (NCT number): NCT04370782, Available From: https://clinicaltrials.gov/ct2/show/NCT0437078 2. 2020.

483. ClinicalTrials.gov identifier (NCT number): NCT04382625, Available From: https://clinicaltrials.gov/ct2/show/NCT0438262 5. 2020.

484. ClinicalTrials.gov identifier (NCT number): NCT04354597, Available From: https://clinicaltrials.gov/ct2/show/NCT0435459 7. 2020.

485. ClinicalTrials.gov identifier (NCT number): NCT04374903, Available From: https://clinicaltrials.gov/ct2/show/NCT0437490 3. 2020.

486. ClinicalTrials.gov identifier (NCT number): NCT04354441, Available From: https://clinicaltrials.gov/ct2/show/NCT0435444 1. 2020.

487. ClinicalTrials.gov identifier (NCT number): NCT04364815, Available From: https://clinicaltrials.gov/ct2/show/NCT0436481 5. 2020.

488. ClinicalTrials.gov identifier (NCT number): NCT04363866, Available From: https://clinicaltrials.gov/ct2/show/NCT0436386 6. 2020.

489. ClinicalTrials.gov identifier (NCT number): NCT04394442, Available From: https://clinicaltrials.gov/ct2/show/NCT0439444 2. 2020.

490. ClinicalTrials.gov identifier (NCT number): NCT04340544, Available From: https://clinicaltrials.gov/ct2/show/NCT0434054 4. 2020.

491. ClinicalTrials.gov identifier (NCT number): NCT04342156, Available From: https://clinicaltrials.gov/ct2/show/NCT0434215 6. 2020.

492. ClinicalTrials.gov identifier (NCT number): NCT04391127, Available From: https://clinicaltrials.gov/ct2/show/NCT0439112 7. 2020.

493. ClinicalTrials.gov identifier (NCT number): NCT04369742, Available From: https://clinicaltrials.gov/ct2/show/NCT0436974 2. 2020.

494. ClinicalTrials.gov identifier (NCT number): NCT04359953, Available From: https://clinicaltrials.gov/ct2/show/NCT0435995 3. 2020.

495. ClinicalTrials.gov identifier (NCT number): NCT04371406, Available From: https://clinicaltrials.gov/ct2/show/NCT0437140 6. 2020.

496. ClinicalTrials.gov identifier (NCT number): NCT04329923, Available From: https://clinicaltrials.gov/ct2/show/NCT0432992 3. 2020 .

497. ClinicalTrials.gov identifier (NCT number): NCT04357808, Available From: https://clinicaltrials.gov/ct2/show/NCT0435780 8. 2020.

498. ClinicalTrials.gov identifier (NCT number): NCT04386239, Available From: https://clinicaltrials.gov/ct2/show/NCT0438623 9. 2020.

499. ClinicalTrials.gov identifier (NCT number): NCT04357860, Available From: https://clinicaltrials.gov/ct2/show/NCT0435786 0.2020.

500. ClinicalTrials.gov identifier (NCT number):NCT04327388, Available From: https://clinicaltrials.gov/ct2/show/NCT0432738 8. 2020. 
501. ClinicalTrials.gov identifier (NCT number): NCT04324073, Available From: https://clinicaltrials.gov/ct2/show/NCT0432407 3. 2020 .

502. ClinicalTrials.gov identifier (NCT number): NCT04332094, Available From: https://clinicaltrials.gov/ct2/show/NCT0433209 4. 2020.

503. ClinicalTrials.gov identifier (NCT number): NCT04332913, Available From: https://clinicaltrials.gov/ct2/show/NCT0433291 3. 2020.

504. ClinicalTrials.gov identifier (NCT number): NCT04377659, Available From: https://clinicaltrials.gov/ct2/show/NCT0437765 9. 2020.

505. ClinicalTrials.gov identifier (NCT number): NCT04331795, Available From: https://clinicaltrials.gov/ct2/show/NCT0433179 5. 2020.

506. ClinicalTrials.gov identifier (NCT number): NCT04345445, Available From: https://clinicaltrials.gov/ct2/show/NCT0434544 5. 2020.

507. ClinicalTrials.gov identifier (NCT number): NCT04359667, Available From: https://clinicaltrials.gov/ct2/show/NCT0435966 7. 2020.

508. ClinicalTrials.gov identifier (NCT number): NCT04335071, Available From: https://clinicaltrials.gov/ct2/show/NCT0433507 1. 2020

509. ClinicalTrials.gov identifier (NCT number): NCT04346355, Available From: https://clinicaltrials.gov/ct2/show/NCT0434635 5. 2020.

510. ClinicalTrials.gov identifier (NCT number): NCT04372186, Available From: https://clinicaltrials.gov/ct2/show/NCT0437218 6. 2020.

511. ClinicalTrials.gov identifier (NCT number): NCT04363736, Available From: https://clinicaltrials.gov/ct2/show/NCT0436373 6. 2020.

512. ClinicalTrials.gov identifier (NCT number): NCT04361032, Available From: https://clinicaltrials.gov/ct2/show/NCT0436103 2. 2020.

513. ClinicalTrials.gov identifier (NCT number): NCT04363853, Available From: https://clinicaltrials.gov/ct2/show/NCT0436385 3. 2020.

514. ClinicalTrials.gov identifier (NCT number): NCT04356937, Available From: https://clinicaltrials.gov/ct2/show/NCT0435693 7. 2020.

515. ClinicalTrials.gov identifier (NCT number): NCT04370834, Available From: https://clinicaltrials.gov/ct2/show/NCT0437083 4. 2020

516. ClinicalTrials.gov identifier (NCT number): NCT04403685, Available From: https://clinicaltrials.gov/ct2/show/NCT0440368 5. 2020.

517. ClinicalTrials.gov identifier (NCT number): NCT04331808, Available From: https://clinicaltrials.gov/ct2/show/NCT0433180 8. 2020.

518. ClinicalTrials.gov identifier (NCT number): NCT04361552, Available From: https://clinicaltrials.gov/ct2/show/NCT0436155 2. 2020.

519. Iranian Clinical Trial Register. Identifier: IRCT20200324046850N2, Available From: https://www.irct.ir/ trial/46713. 2020.

520. Iranian Clinical Trial Register. Identifier: IRCT20200325046859N1, Available From: https://www.irct.ir/ trial/46811. 2020.

521. Iranian Clinical Trial Register. Identifier: IRCT20200328046886N1, Available From: https://www.irct.ir/ trial/46885. 2020.
522. Iranian Clinical Trial Register. Identifier: IRCT20200418047126N1, Available From: https://www.irct.ir/ trial/47707. 2020.

523. Iranian Clinical Trial Register. Identifier: IRCT20200421047155N1, Available From: https://www.irct.ir/ trial/47387. 2020.

524. Iranian Clinical Trial Register. Identifier: IRCT20200422047168N1, Available From: https://en.irct.ir/ trial/47437. 2020.

525. Iranian Clinical Trial Register. Identifier: IRCT20200325046859N2, Available From: https://www.irct.ir/ trial/47200. 2020.

526. Iranian Clinical Trial Register. Identifier: IRCT20190122042450N4, Available From: https://www.irct.ir/ trial/47090. 2020.

527. Iranian Clinical Trial Register. Identifier: IRCT20151222025660N2, Available From: https://www.irct.ir/ trial/47319. 2020.

528. Iranian Clinical Trial Register. Identifier: IRCT20200406046968N1, Available From: https://www.irct.ir/ trial/47021. 2020.

529. Iranian Clinical Trial Register. Identifier: IRCT20151227025726N13, Available From: https://www.irct. $\mathrm{ir} / \mathrm{trial} / 46681.2020$.

530. Iranian Clinical Trial Register. Identifier: IRCT20200510047383N1, Available From: https://www.irct.ir/ trial/48024. 2020.

531. Iranian Clinical Trial Register. Identifier: IRCT20150303021315N17, Available From: https://www.irct. ir/trial/46550. 2020.

532. Iranian Clinical Trial Register. Identifier: IRCT20200511047396N1, Available From: https://www.irct.ir/ trial/48048. 2020

533. Iranian Clinical Trial Register. Identifier: IRCT20190804044429N1, Available From: https://www.irct.ir/ trial/46741. 2020

534. Iranian Clinical Trial Register. Identifier: IRCT20080901001165N53, Available From: https://www.irct. ir/trial/48066. 2020

535. Iranian Clinical Trial Register. Identifier: IRCT20100228003449N27, Available From: https://www.irct. ir/trial/46545. 2020.

536. Iranian Clinical Trial Register. Identifier: IRCT20100228003449N28, Available From: https://www.irct. ir/trial/46538. 2020.

537. Iranian Clinical Trial Register. Identifier: IRCT20150914024017N1, Available From: https://www.irct.ir/ trial/46665. 2020.

538. Iranian Clinical Trial Register. Identifier: IRCT20200406046968N3, Available From: https://www.irct.ir/ trial/47152. 2020.

539. ClinicalTrials.gov identifier (NCT number): NCT04348071, Available From: https://clinicaltrials.gov/ct2/show/NCT0434807 1. 2020.

540. ClinicalTrials.gov identifier (NCT number): NCT04381936, Available From: https://clinicaltrials.gov/ct2/show/NCT0438193 6 ? term $=$ NCT0 $381936 \&$ cond $=$ COVID $-19 \&$ draw $=2 \&$ rank $=1$. 2020 .

Publisher's Note Springer Nature remains neutral with regard to jurisdictional claims in published maps and institutional affiliations. 